PASJ: Publ. Astron. Soc. Japan , 1-??,

(C) 2021. Astronomical Society of Japan.

\title{
Luminosity effect of O I 7771-5 triplet and atmospheric microturbulence in evolved A-, F-, and G-type stars
}

\author{
Yoichi TAKEDA, ${ }^{1,2}$ Gwanghui JeOng, ${ }^{3,4}$ and Inwoo Han, ${ }^{3,4}$ \\ ${ }^{1}$ National Astronomical Observatory, 2-21-1 Osawa, Mitaka, Tokyo 181-8588, Japan \\ ${ }^{2}$ SOKENDAI, The Graduate University for Advanced Studies, 2-21-1 Osawa, Mitaka, Tokyo 181-8588 \\ takeda.yoichi@nao.ac.jp \\ ${ }^{3}$ Korea Astronomy and Space Science Institute, 776, Daedeokdae-Ro, Youseong-Gu, Daejeon 34055, Korea \\ ${ }^{4}$ Korea University of Science and Technology, 217, Gajeong-ro Yuseong-gu, Daejeon 34113, Korea \\ tlotv@kasi.re.kr,iwhan@kasi.re.kr
}

(Received 2017 October 23; accepted 2017 December 13)

\begin{abstract}
It is known that the strength of neutral oxygen triplet lines at $7771-5 \AA$ shows a luminosity effect in evolved A through G stars. However, its general behavior across the HR diagram is not yet well understood, since the applicability limit of the relations proposed by various previous work (tending to be biased toward supergiants) still remains unclear. Besides, our understanding on the nature of atmospheric micro-scale turbulence, which is considered to play a significant role (along with the non-LTE line intensification) for the cause of this effect, is still insufficient. Towards clarifying these problems, we carried out an extensive non-LTE spectrum-fitting analysis of $\mathrm{O}$ I $7771-5$ lines for unbiased sample of 75 evolved A-, F,- and G-type stars over wide luminosity classes (from subgiants through supergiants) including rapid rotators, from which the total equivalent width $\left(W_{77}\right)$ was derived and the microturbulence $(\xi)$ was determined by two different (profile- and abundance-based) methods for each star. While we confirmed that $W_{77}$ tends to increase in the global sense as a star's absolute magnitude $\left(M_{V}\right)$ becomes more luminous, distinctly different trends were found between lower-gravity $(\log g \lesssim 2.5)$ and higher-gravity $(\log g \gtrsim 2.5)$ stars, in the sense that the $M_{V}$ vs. $W_{77}$ formulas proposed by past studies are applicable only to the former supergiant group. In case of using $W_{77}$ for empirical $M_{V}$ evaluation by such simple formulas, it is recommended to confine only to supergiants of $-5 \gtrsim M_{V} \gtrsim-10$. Regarding the microturbulence significantly controlling $W_{77}$, it roughly shows an increasing tendency with a decrease in surface gravity. However, the trend is not monotonic but rather intricate (e.g., hump, stagnation, or discontinuously large increase) depending on the stellar type and evolutionary stage.
\end{abstract}

Key words: line: profiles — stars: atmospheres — stars: fundamental parameters — stars: evolution - turbulence

\section{Introduction}

Ever since the historical work of old days (Merrill 1925, Keenan \& Hynek 1950), the triplet lines of neutral oxygen at $7771.94,7774.17$, and $7775.4 \AA\left(3 \mathrm{~s}{ }^{5} \mathrm{~S}^{\mathrm{o}}-3 \mathrm{p}{ }^{5} \mathrm{P}\right.$, multiplet 1 ) are known to be of considerable strength and easily measurable in the spectra of A-, F-, and G-type stars. In particular, an important aspect related to this feature is the luminosity effect; i.e., its strength (herein referred to as $W_{77}$, which is the total integrated equivalent width of the whole triplet) tends to progressively grow as a star becomes more luminous. It is natural to come up with a possibility to make use of this characteristic to estimate the stellar absolute magnitude $\left(M_{V}\right)$ by simply measuring $W_{77}$. Accordingly, these triplet lines inspired the interest of a number of astrophysicists, who investigated the nature of $M_{V}$ vs. $W_{77}$ relation and established useful analytical formulas, as summarized in table $1 .^{1}$

Unfortunately, our understanding of these $M_{V}$ vs. $W_{77}$

The references listed in table 1 were selected by consulting the literature in the recent papers of Kovtyukh, Gorlova, and Belik relations proposed by various investigators is not yet sufficient, since it appears still uncertain to which luminosity ranges such simple relations are applicable.

- For example, Arellano Ferro, Giridhar, and Rojo Arellano (2003; the latest of the series of papers published by their group) reported that $M_{V}$ can be well represented by a quadratic polynomial of $W_{77}$ (with remarkable accuracies of only $\pm 0.38 \mathrm{mag}$ ) for evolved A-, F, and Gstars over a wide luminosity range (from $M_{V} \sim 0$ mag to -9 mag; cf. their Fig. 5b), in which even the color term $\left[(b-y)_{0}\right]$ has been omitted (which was included in their earlier papers but concluded to be unnecessary). If this formula is valid, it must be very useful.

- However, according to the recent investigation by Kovtyukh, Gorlova, and Belik (2012), $M_{V}$ and $W_{77}$ do not seem to follow a simple and smooth one-to-one correspondence but show a considerable scatter, especially the lower luminosity region of $0 \gtrsim M_{V} \gtrsim-3$ (cf. their Fig. 2).

(2012) as well as Dambis (2013). They are not meant to be complete. 
Although they tried reproduce their observational $M_{V}$ vs. $W_{77}$ data by an analytical relation including terms involving $T_{\text {eff }}$ (effective temperature), $\log g$ (surface gravity), $\xi$ (microturbulence), and $[\mathrm{Fe} / \mathrm{H}]$ (metallicity), such a complex formula is not practically useful as long as empirical determination of $M_{V}$ is concerned.

Looking over the various literature on the luminosity effect of O I 7771-5 (table 1), we note that, while intrinsically bright "supergiants" were preferably investigated in almost all work, less attention has been paid to "giants" which are comparatively less luminous. Accordingly, it seems necessary to have a better understanding of the behavior of $\mathrm{O}$ I $7771-5$ strength for $\mathrm{A}-\mathrm{G}$ giants as well as supergiants, before we can answer the question "Is $M_{V}$ simply a function of $W_{77}$ as reported by Arellano Ferro et al. (2003)?" or "Does it actually depend not only $W_{77}$ but also on other stellar parameters?"

Motivated by this situation, we decided to carry out a comprehensive study on the strength of $\mathrm{O}$ I $7771-5$ lines for evolved A-, F-, and G-type stars of various luminosity classes (subgiants, giants, and supergiants) based on the high-dispersion spectra of an unbiased sample of 75 stars observed at Bohyunsan Astronomical Observatory, in order to clarify the parameter dependence of $W_{77}$ across the HR diagram. Unlike previous investigations, we make use of the synthetic spectrum-fitting technique for evaluation of $W_{77}$; i.e., $W_{77}$ is inversely computed from the oxygen abundance solution accomplishing the best fit. This approach is particularly effective for rapid rotators (occasionally seen in $\mathrm{A}-\mathrm{F}$ giants), for which direct measurement of $W_{77}$ is not easy because of the contamination of other lines. This is the primary purpose of this study.

Besides, as a by-product resulting from the analysis, we can study the behavior of microturbulence ${ }^{2}(\xi)$ over a wide parameter range of evolved $A-G$ stars, which is considered to be an important parameter affecting $W_{77}$, because O I 7771-5 lines are strong and saturated in these stars. We will derive this key parameter by two (profilebased and abundance-based) methods; these two kinds of $\xi$ values determined by independent techniques would make a useful comparison. In this context, Takeda (1992) previously studied how $\xi$ behaves in $\mathrm{A}-\mathrm{F}$ stars of various luminosity classes, while comparing the observed $W_{77}$ data taken from various literature with the theoretically calculated non-LTE equivalent widths. While the global nature of $\xi$ was roughly elucidated in that paper, $\xi$ values of individual stars could not be discussed in terms of their dependence upon stellar parameters. Accordingly, this is a good opportunity to challenge the task which was left undone in Takeda (1992). Especially, since studies on the behavior of $\xi$ for $\mathrm{A}-\mathrm{F}$ giants of luminosity class III seem

2 This is by definition the microscopic turbulent velocity dispersion, the characteristic scale of which is assumed to be much smaller than the photon mean-free-path. Accordingly, it is formally included into the Doppler width of line-opacity profile in parallel with the velocity of thermal motion. While the strength of a weak line (on the linear part of the curve of growth) is hardly affected by this parameter, that of a strong saturated line (on the flat part of the curve of growth) is very sensitive to it. to have been barely done (see, e.g., Fig. 11 of Gray 1978), presumably because of the existence of rapid rotators, our analysis would make a new contribution to this field. As such, this checking upon $\xi$ defines the second aim of this paper.

\section{Observational Data}

The targets of this study are 75 A-, F-, and G-type stars (from subgiants through supergiants), which had already evolved off the main sequence. The basic data of these objects are given in table 2. (See also tableE.dat given as online material for more detailed information.)

The observations of these objects (except for HD 20902) were carried out on 2012 October 6-7, 2013 March 2829, 31, and 2013 May 21-23 by using BOES (Bohyunsan Observatory Echelle Spectrograph) attached to the $1.8 \mathrm{~m}$ reflector at Bohyunsan Optical Astronomy Observatory. Using a $2 \mathrm{k} \times 4 \mathrm{k}$ CCD (pixel size of $15 \mu \mathrm{m} \times 15 \mu \mathrm{m}$ ), this echelle spectrograph enabled us to obtain spectra of wide wavelength coverage (from $\sim 3600 \AA$ to $\sim 9200 \AA$ ) at a time. We used $200 \mu \mathrm{m}$ fiber corresponding to the resolving power of $R \simeq 45000$. The integrated exposure time for each star was typically about ten to several tens minutes. The reduction of the echelle spectra (bias subtraction, flat fielding, scattered-light correction, spectrum extraction, wavelength calibration, co-addition of spectra to increase $\mathrm{S} / \mathrm{N}$, and continuum normalization) was carried out by using the "echelle" package of the software $\mathrm{IRAF}^{3}$ in the standard manner. For most of the targets, we could accomplish sufficiently high $\mathrm{S} / \mathrm{N}$ ratio (typically a few hundreds) at the orange-red region relevant to the present study. Regarding HD 20902 ( $\alpha$ Per), we exceptionally used the high-dispersion spectrum (also obtained by using BOES) published by Lee et al. (2006).

\section{Stellar Parameters}

The parameters of 75 program stars were determined by the photometric data (apparent magnitude $V$ and $B-V$ color) taken from the Hipparcos catalogue (ESA 1997 ) and the newly reduced Hipparcos parallaxes $\pi$ (van Leeuwen 2007). ${ }^{4}$ We estimated the interstellar extinction $\left(A_{V}\right)$ for each star by using Hakkila et al.'s (1997) EXTINCT program ${ }^{5}$ from the galactic coordinates $(l, b)$ along with the distance $d(\propto 1 / \pi)$; and the color excess was derived as $E_{B-V}=A_{V} / 3.0$. Then, Alonso, Arribas, and Martínez-Roger's (1999) Eq.(3) (for $B-V<0.75$ )

3 IRAF is distributed by the National Optical Astronomy Observatories, which is operated by the Association of Universities for Research in Astronomy, Inc. under cooperative agreement with the National Science Foundation.

4 Although we basically adopted the new-reduction data published by van Leeuwen (2007), the first-released Hipparcos parallaxes (ESA 1997) were exceptionally used for 8 stars as remarked in table 2 (for which the values of two catalogues are appreciably different because of being distant) by considering the consistency between the resulting $T_{\text {eff }} / \log g$ and the spectral type/luminosity class.

5 Available at $\langle$ http://asterisk.apod.com/library/ASCL/extinct/extinct.for $\rangle$. 
and Eq. (4) (for $B-V \geq 0.75$ ) were invoked to derive $T_{\text {eff }}$ from the reddening-corrected color $B-V$, where we assumed $[\mathrm{Fe} / \mathrm{H}]=0$. Further, the absolute magnitude $\left(M_{V}\right)$ and bolometric luminosity $(L)$ were calculated from the extinction-corrected $V$, parallax $(\pi)$, and the bolometric correction (B.C.) estimated with the help of Alonso et al.'s (1999) Eq. (17) and Eq. (18).

Here, we should keep in mind that Alonso et al.'s (1999) analytical formulas for $T_{\text {eff }}$ and B.C. were derived specifically for class-III giants (besides, $T_{\text {eff formula is applicable }}$ for $T_{\text {eff }} \lesssim 8000 \mathrm{~K}$, while B.C formula is for $T_{\text {eff }} \lesssim 9100 \mathrm{~K}$ ). Since we universally applied them to our program stars of $\mathrm{A}-\mathrm{F}-\mathrm{G}$ type covering wide range of luminosity classes (subgiants through supergiants), errors caused by extrapolation may be more or less expected especially for the case of supergiants. In order to examine this point, we compared these Alonso et al.'s formula with Flower's (1996) calibration for supergiants of wide $T_{\text {eff }}$ range (cf. Table 4 therein), and found that the differences are unimportant $\left(\lesssim 100-300 \mathrm{~K}\right.$ in $T_{\text {eff }}$ or $\lesssim$ a few hundredths mag in B.C.) at $B-V \gtrsim 0.1$, while the discrepancies begin to manifestly grow once $B-V$ becomes less than 0.1 (e.g., up to $\sim 1000 \mathrm{~K}$ in $T_{\text {eff }}$ and $\sim 0.2-0.3 \mathrm{mag}$ in B.C. at $B-V \sim 0$ ) in the sense that Alonso et al.'s formula underestimates both $T_{\text {eff }}$ and |B.C.|. Accordingly, we may state that inadequate parameters may possibly result by our application of Alonso et al.'s relations for the case of A-type (especially early-A) supergiants. Yet, since the relevant stars of this type in our sample are distant and confined to the Galactic plane, their stellar parameters tend to be unreliable by themselves in any case, due to considerable ambiguities in parallax as well as interstellar extinction (see subsection 7.1).

The $\log L$ vs. $\log T_{\text {eff }}$ diagram of the program stars are depicted in figure 1 , where the evolutionary tracks (for the solar metallicity and corresponding stellar masses of $M \sim 1.5-20 M_{\odot}$ ) computed by Lejeune and Schaerer (2001) are also shown for comparison. Making use of the fact that these evolutionary tracks in figure 1 tend to run almost horizontally (i.e., $L$ is quite sensitive to $M$ but rather inert to $T_{\text {eff }}$ ), we assume that $M$ is a monotonic function of $L$ for any given $T_{\text {eff }}$ at $10000 \mathrm{~K} \gtrsim T_{\text {eff }} \gtrsim 5000 \mathrm{~K}$, where we define this function by numerically interpolating the light-green portions of the tracks in figure 1. In this way, we could evaluate $M$ (from $T_{\text {eff }}$ and $L$ ), from which the surface gravity $(\log g)$ was further derived by the relation $g \propto T_{\mathrm{eff}}^{4} M / L$. The finally resulting values of $M_{V}, L, M, T_{\text {eff }}$, and $\log g$ for each star are summarized in table 2; more detailed data (including the basic photometric data, parallax, interstellar extinction, bolometric correction, etc) are presented in the online material (tableE.dat).

These $T_{\text {eff }}$ and $\log g$ values of 75 program stars determined by rather rough methods are compared with available literature data (taken from the SIMBAD database) in figures $2 \mathrm{a}$ and $2 \mathrm{~b}$, respectively, where we can see a reasonable consistency for $T_{\text {eff }}$, while some systematic trend (though not so serious) is observed for $\log g$. The correlations of $T_{\text {eff }}$ vs. spectral type and $\log g$ vs. luminosity class are depicted in figures $2 \mathrm{c}$ and $2 \mathrm{~d}$, respectively.

It may be worth commenting on the accuracy of useful analytical relations. The basic $M$ vs. $L$ relations we employed for deriving $M$ (from $L$ ) are displayed in figure $3 \mathrm{a}$ for three values of $T_{\text {eff }}(5000,7500,10000 \mathrm{~K})$, which are quite similar to each other reflecting the nearhorizontal nature of evolutionary tracks (i.e., insensitive to changes in $\left.T_{\text {eff }}\right)$. The $L$ values for individual stars are plotted against the resulting $M$ in figure $3 \mathrm{~b}$. Since $\log L$ vs. $\log M$ relation almost follows the straight line $\left[\log \left(L / L_{\odot}\right)=0.194+4.07 \log \left(M / M_{\odot}\right)\right.$ according to the linear-regression analysis], this means that the power law of $L \propto M^{4}$ holds fairly well. Actually, the mutual relation between $\log g, M_{\mathrm{bol}}$, and $T_{\text {eff }}$ for $\mathrm{A}-\mathrm{F}$ stars proposed by Takeda (1992) (later, the constant was slightly revised by Takeda and Takada-Hidai 1994)

$$
\log g=0.30 M_{\mathrm{bol}}+4 \log T_{\text {eff }}-12.05
$$

as well as the equation for the stellar mass adopted by Takeda and Takada-Hidai (1994) for A-F supergiants

$$
\log \left(M / M_{\odot}\right)=\left[4 \log \left(T_{\text {eff }} / T_{\text {eff }, \odot}\right)-\log \left(g / g_{\odot}\right)\right] / 3
$$

are both based on the scaling law of $L \propto M^{\alpha}$ with $\alpha=4.0$. In figures $3 \mathrm{c}$ and $3 \mathrm{~d}$ are compared the finally adopted values of $M_{\mathrm{bol}}$ and $M$ with those derived by these simple analytical formulas, respectively. We see from figure $3 \mathrm{c}$ that equation (1) is sufficiently accurate (differences of $M_{\text {bol }}$ are only $\left.\lesssim 0.2 \mathrm{mag}\right)$, while application of equation (2) somewhat overestimates the stellar mass by $\sim 0.05^{-}$ $0.1 \operatorname{dex}(\lesssim 20 \%)$.

\section{Model Atmospheres and Non-LTE Calculations}

The model atmosphere for each star was constructed by two-dimensionally interpolating Kurucz's (1993) ATLAS9 model grid (models with convective overshooting) with respect to $T_{\text {eff }}$ and $\log g$ determined in section 3 , where we exclusively applied the solar-metallicity models.

In order to adequately incorporate the non-LTE effect, which is requisite for the $\mathrm{O}$ I triplet lines under study, we carried out non-LTE calculations for oxygen on an extensive grid of solar-metallicity model atmospheres resulting from combinations of eleven $T_{\text {eff }}$ values $(5500,6000,6500$, $7000,7500,8000,8000,8500,9000,9500,10000 \mathrm{~K})$ and six $\log g$ values $(1.5,2.0,2.5,3.0,3.5,4.0),{ }^{6}$ and eleven $\xi$ values $\left(0,1,2,3,4,5,6,7,8,9,10 \mathrm{~km} \mathrm{~s}^{-1}\right)$, which almost cover the parameter ranges of our program stars. See Takeda (2003) and the references therein for details of the calculation procedures. Regarding the treatment of collisional rates with neutral hydrogen atoms, we adopted the conventional treatment without any corrections $(k=1)$. The non-LTE departure coefficients $[b(\tau)]$ applied to each star were then derived by interpolating this grid in terms of $T_{\text {eff }}$ and $\log g$, as was done for model atmospheres. Since

$6 \quad$ Since ATLAS9 model grid does not include $\log g=1.5$ models at $T_{\text {eff }} \geq 9500 \mathrm{~K}$ presumably because of an instability problem, models of only five gravities $(\log g=2.0,2.5,3.0,3.5$, and 4.0) could be used for $T_{\text {eff }}=9500$ and $10000 \mathrm{~K}$. 
departure coefficients are not sensitive to a change of $\xi$, an appropriate dataset of the grid near to the relevant $\xi$ value was employed.

\section{Spectrum Fitting of O I 7771-5}

Since the spectra of A-F-G stars in the $7765-7785 \AA$ region (comprising O I 7771-5 triplet lines and Fe I 7781 line) depend on various factors ( $\mathrm{O}$ and $\mathrm{Fe}$ abundances, microturbulence, macroturbulence/rotational velocity, radial velocity, etc.), we may obtain information of these parameters by a careful analysis based on the spectrum synthesis technique, as previously done by Takeda and Sadakane (1997) for A-F stars in the Hyades cluster.

Accordingly, we searched for the solutions for the oxygen abundance $(A(\mathrm{O}))$, Fe abundance $(\mathrm{A}(\mathrm{Fe}))$, microturbulence $\left(\xi_{\mathrm{p}}\right),{ }^{7}$ macrobroadening velocity $\left(v_{\mathrm{M}}\right)$, and radial velocity $\left(V_{\mathrm{rad}}\right)$ which accomplish the best fit (minimizing $O-C$ residuals) between the theoretical and observed spectrum in the $7765-7785 \AA$ region, by applying the automatic fitting algorithm described in Takeda (1995). Regarding the macrobroadening function (to be convolved with the intrinsic profile), we adopted the classical rotational broadening function (see, e.g., Gray 2005) with the limb-darkening coefficient of $\epsilon=0.5$. Therefore, $v_{\mathrm{M}}$ may be regarded as equivalent to $v_{\mathrm{e}} \sin i$ (projected rotational velocity) if macroturbulence is negligible. Generally, if $v_{\mathrm{M}}$ is larger than several tens $\mathrm{km} \mathrm{s}^{-1}$, we may safely consider $v_{\mathrm{M}} \simeq v_{\mathrm{e}} \sin i$; otherwise, appreciable contribution of macroturbulence to $v_{\mathrm{M}}$ (in addition to $v_{\mathrm{e}} \sin i$ ) is quite probable. As to the atomic data of spectral lines, we exclusively consulted the compilation by Kurucz and Bell (1995). The data for the three O I 7771-5 lines are summarized in table 3. Although all atomic lines given in their compilation for this wavelength region were included in the spectrum synthesis, the abundances of elements other than $\mathrm{O}$ and Fe were fixed at the solar abundances.

The convergence of the solutions was satisfactory for most cases. ${ }^{8}$ Nevertheless, we sometimes encountered instability or divergence of solutions; in such cases, we had to fix the relevant parameter at an appropriate value. The resulting velocity parameters $\left(v_{\mathrm{M}}\right.$ and $\left.\xi_{\mathrm{p}}\right)$ for each star are summarized in table 2. It is demonstrated in figure 4 that the theoretical spectra for the converged solutions properly fit with the observed spectra.

We then inversely computed the equivalent width $\left(W_{77}\right)$ for the whole $\mathrm{O}$ I $7771-5$ triplet by using the finally converged solutions of $A(\mathrm{O})$ and $\xi_{\mathrm{p}}$ along with the same atomic data and model atmosphere used in the fitting analysis. For this purpose, we used Kurucz's (1993) WIDTH9 program, which was considerably modified in

$7 \quad$ Hereinafter, we refer to this parameter as $\xi_{\mathrm{p}}$ ("p" means "profile") because this is the microturbulence derived from line profiles.

8 Practically, some tricks are needed in order to accomplish successful convergence for all parameters. For example, iteration should be done by fixing $\xi$ at the first round, followed by the second round of iteration (starting from converged solution in the first round) where $\xi$ is allowed to vary toward convergence. many respects (e.g., to incorporate the non-LTE effect, to allow complex feature comprising a multiple of line components, etc.). The $W_{77}$ values finally obtained as such, based on which we will discuss the luminosity effect of $\mathrm{O}$ I 7771-5 lines, are given in table 2 .

\section{Abundance-Based Microturbulence}

Although we derived the microturbulence $\left(\xi_{\mathrm{p}}\right)$ from the profile of $\mathrm{O}$ I $7771-5$ triplet in section 5 , this is not the usual approach. The conventional method of determining this parameter is to require the consistency of abundances derived from strong and weak lines, which makes use of the fact that the former abundance is much more $\xi$-sensitive than the latter. It is meaningful to determine also this $\xi_{\mathrm{a}}{ }^{9}$ and see how these two kinds of microturbulence are compared with each other. For this purpose, we use O I 6155-8 lines $\left(3 \mathrm{p}^{5} \mathrm{P}-4 \mathrm{~d}^{5} \mathrm{D}^{\mathrm{o}}\right.$, multiplet 10$)$, which are much weaker than O I 7771-5 lines and thus suitable. Although the orange region where these lines situate is more crowded with other spectral lines compared to the case of O I 7771-5, oxygen abundance can be determined (even for rapid rotators) by applying the spectrum-synthesis technique, as recently done by Takeda, Hashimoto, and Honda (2017) for their study of F-G type stars in the Pleiades cluster.

In almost the same manner as done in section 5, we conducted a spectrum-fitting analysis in the $6143-6168 \AA$ region, while changing the abundances of $\mathrm{O}, \mathrm{Na}, \mathrm{Si}, \mathrm{Ca}$, and Fe to search for the best-fit solution (microturbulence was fixed at $\left.\xi_{\mathrm{p}}\right)$. The finally accomplished fit between the theoretical and observed spectra are demonstrated in figure 5 . Then, the equivalent width $\left(W_{61}\right)$ corresponding to whole O I 6155-8 was evaluated from the converged solutions of $A(\mathrm{O})$ and $\xi_{\mathrm{p}}$ by integrating the synthesized spectrum of 9 component lines (cf. table 3) as done in section 5 for $W_{77}$.

We computed the non-LTE oxygen abundances $\left(A_{77}^{\mathrm{N}}\right.$ and $A_{61}^{\mathrm{N}}$ ) (and also the LTE abundances $A^{\mathrm{L}}$ as well as the non-LTE correction $\Delta \equiv A^{\mathrm{N}}-A^{\mathrm{L}}$ ) from such established $W_{77}$ and $W_{61}$ for each star while progressively changing the $\xi$ values. Based on this set of abundances, $\xi_{a}$ may be defined as the $\xi$ value satisfying $A_{77}^{\mathrm{N}}=A_{61}^{\mathrm{N}}$. Although this attempt was not always successful, we could determine $\xi_{\text {a }}$ for 62 stars (cf. table 2), which will be discussed in subsection 7.4 in comparison with $\xi_{\mathrm{p}}$. Figure 6 shows the $T_{\text {eff-dependence of equivalent widths }}(W)$, microturbulence $\left(\xi_{\mathrm{a}}\right)$, non-LTE abundances $\left(A^{\mathrm{N}}\right)$ as well as nonLTE corrections $(\Delta)$ corresponding to $\xi_{\mathrm{a}}$. The complete data of $W, A(\mathrm{O})$, and $\Delta$ for both line features are presented in tableE.dat of the online material.

\footnotetext{
9 Hereinafter, we refer to this parameter as $\xi_{\mathrm{a}}$ ("a" means "abundance") because this is the microturbulence derived by requiring the abundance consistency between lines of different strengths.
} 


\section{Discussion}

\subsection{Accuracy of absolute magnitude and equivalent width}

Since the main purpose of this paper concerns the empirical relation between $M_{V}$ and $W_{77}$, it may be appropriate here to mention the accuracies of these quantities (cf. table 2) which we derived in section 3 and section 5 .

Regarding $M_{V}$, two factors are involved in its uncertainty: (i) error $\left(\sigma_{\pi}\right)$ in the Hipparcos parallax $(\pi)$, which contributes to an error in $M_{V}$ by the relation $\delta M_{V}^{\pi} \simeq$ $5 \log \left(1+\sigma_{\pi} / \pi\right)$, and (ii) error in the adopted interstellar extinction $\left(\delta M_{V}^{A}=\sigma_{A}\right)$, which is estimated by the standard deviation of $A_{V}$ resulting from the EXTINCT program. Then, the total error of $M_{V}$ may be evaluated by the square-sum-root of these two as $\delta M_{V}^{\pi+A} \equiv$ $\sqrt{\left(\delta M_{V}^{\pi}\right)^{2}+\left(\delta M_{V}^{A}\right)^{2}}$. We plot such evaluated $\delta M_{V}^{\pi}, \delta M_{V}^{A}$, and $\delta M_{V}^{\pi+A}$ for eah star against $M_{V}$ in figure $7 \mathrm{a}, 7 \mathrm{~b}$, and $7 \mathrm{c}$, respectively. We can see the following characteristics from these figures.

- Both $\delta M_{V}^{\pi}$ and $\delta M_{V}^{A}$ tend to increase as $M_{V}$ becomes brighter, which simply reflects the fact that brighter $M_{V}$ stars are generally more distant and thus suffer larger errors in $\pi$ as well as in $A_{V}$.

- There is a tendency that the extent of $\delta M_{V}^{\pi}$ is larger than that of $\delta M_{V}^{A}$ (especially for brighter $M_{V}$ stars), which means that parallax error is comparatively more important in affecting the total uncertainty of $M_{V}$.

- According to figure $7 \mathrm{c}$, the error of $M_{V}$ is typically several tenths of magnitude for giants $\left(M_{V} \gtrsim-2\right)$, while it tends to suddenly grow with luminosity at $-2 \lesssim M_{V}$ and even attains as much as $\sim 1$ mag at $M_{V} \sim-5$.

— These intrinsically luminous supergiants (around $M_{V} \sim$ -5) with considerable $M_{V}$ errors have luminosities around $\log \left(L / L_{\odot}\right) \sim 4-5$. Since such stars tend to be of rather high $T_{\text {eff }}(\gtrsim 8000 \mathrm{~K})$ as seen from figure 1 , we should bear in mind that our $M_{V}$ values of A-type supergiants may suffer rather large uncertaities.

On the other hand, we may expect that $W_{77}$ could be established with a rather high precision, because it was derived by applying the spectrum-fitting technique, where a sufficiently good fit could be accomplished by the theoretical synthesized spectrum regardless of how complex the line profile is.

Regarding the error of equivalent width $(W)$ stemming from the spectrum noise, we may invoked the formula derived by Cayrel (1988)

$$
\delta W \simeq 1.6(w \delta x)^{1 / 2} \epsilon,
$$

where $\delta x$ is the pixel size (or sampling step), $w$ is the full-width at half maximum, and $\epsilon \equiv(\mathrm{S} / \mathrm{N})^{-1}$. Putting $w \simeq 7773 v_{\mathrm{M}} / c$ ( $w$ is in $\AA$ and $c$ is the velocity of light $), \delta x \simeq$ $0.04 \AA$, and $\mathrm{S} / \mathrm{N} \sim 200$ (typical value), we obtain $\delta W \sim$ $(1 / 1000) \sqrt{v_{\mathrm{M}} / 10}$ (where $\delta W$ is in $\AA$ and $v_{\mathrm{M}}$ is in $\mathrm{km} \mathrm{s}^{-1}$ ). In case of small $v_{\mathrm{M}}$ where the three component lines are clearly split, we may regard $W \simeq W_{77} / 3$, while $W=W_{77}$ for the case of merged triplet with large $v_{\mathrm{M}}$. Considering four representative cases resulting from combinations of
$v_{\mathrm{M}}=10$ and $100 \mathrm{~km} \mathrm{~s}^{-1}$ and $W_{77}=0.5$ and $1 \AA$, we can conclude that $\delta W / W$ is $<1 \%$ in any event, which is negligibly insignificant.

For reference, we also compare our $M_{V}$ and $W_{77}$ values with those published by Arellano Ferro et al. (2003; 6 stars in common; mean equivalent widths designated as " $W_{74,0}$ " in their Table 2 were adopted for four Cepheids) as well as Kovtyukh et al. (2012; 11 stars in common; "literature $M_{V}$ " given in their Table 1 were used) in figure $7 \mathrm{~d}$ and figure $7 \mathrm{e}$, respectively, where we can see a reasonable consistency without any systematic difference.

\subsection{Observed behavior of W(O I r7r1-5)}

We now discuss how $W_{77}$ depends on the stellar parameters, especially in terms of the absolute magnitude. In figure 8 are plotted the resulting $W_{77}$ values of 75 program stars against $T_{\text {eff }}, \log g, v_{\mathrm{M}}$, and $M_{V}$.

In figure $8 \mathrm{a}$, the triplet line strengths for 46 A-type dwarfs (which were inversely computed from the $\mathrm{O}$ abundance results of Takeda et al. 2008a) as well as those of 160 FGK dwarfs (which were obtained by summingup the observed equivalent widths of three lines published by Takeda \& Honda 2005) are also shown for comparison (note that oxygen-deficient stars are included in these samples of main-sequence stars). Immediately noticeable from figure $7 \mathrm{a}$ is the increasing tendency toward higher $T_{\text {eff }}$ (especially from $G$ to $F$ ), which is 8ctually an expected trend for such high-excitation lines (cf. figure 9a). The fact that $W_{77}$ values of almost all evolved stars of our sample surpass those of main-sequence stars at any given $T_{\text {eff }}$ evidently indicates that O I $7771-5$ triplet generally strengthens as a result of stellar evolution.

Regarding the $W_{77}$ vs. $v_{\mathrm{M}}$ plot shown in figure $8 \mathrm{c}$, we see a correlation that $W_{77}$ steeply grows with an increase in $v_{\mathrm{M}}$ for $\log g<2.5$ stars (open circles) at comparatively low $v_{\mathrm{M}}$ values being less than several tens $\mathrm{km} \mathrm{s}^{-1}$. Recalling that appreciable contribution of macroturbulence (or almost dominated by the macroturbulence) is expected for $v_{\mathrm{M}}$ of such stars (cf. section 5), this prominent growth of $W_{77}$ with $v_{\mathrm{M}}$ is attributed to the increase of $\xi$ (compare figure 6a with figure $6 \mathrm{~b}$ ), because microand macro-turbulence are likely to be closely connected. Meanwhile, no clear $v_{\mathrm{M}}$-dependence is observed in $W_{77}$ for stars with larger values of $v_{\mathrm{M}}$, where $v_{\mathrm{M}}$ is essentially equivalent to $v_{\mathrm{e}} \sin i$.

Since surface gravity and absolute magnitude are intimately related with each other as indicated by equation (1), it is natural that $W_{77}$ vs. $\log g$ plot (figure 8b) and $W_{77}$ vs. $M_{V}$ plot (figure $8 \mathrm{~d}$ ) look quite similar. It is figure $8 \mathrm{~d}$ that reveals the nature of the luminosity effect for $\mathrm{O}$ I $7771-5$ in evolved $\mathrm{A}-\mathrm{G}$ stars. We note the following characteristics from this figure.

- We can observe that lower gravity stars of $\log g<2.5$ (open circles) and higher gravity stars of $\log g>2.5$ (filled circles) show apparently different behaviors of $W_{77}$.

- Remarkably, although the various empirical $W_{77}$ vs. $M_{V}$ formulas proposed so far (gray lines) are more or 
less consistent with the distribution of former lowergravity group (supergiants) at the wide $M_{V}$ range $\left(1 \gtrsim M_{V} \gtrsim-8\right)$, they never represent that of the latter higher-gravity group (subgiants, giants).

- We should be careful for the stars of $0 \gtrsim M_{V} \gtrsim-5$, in which these two groups are mixed; i.e., lower $T_{\text {eff }} /$ lower $\log g(<2.5)$ stars and higher $T_{\text {eff }} /$ higher $\log g(>2.5)$ stars. Again, the published formulas summarized in table 1 are relevant only for the former (F-G supergiants) while not for the latter (Atype giants).

- The trend of $W_{77}$ vs. $M_{V}$ for the higher-gravity $(\log g>2.5)$ group (red lines depicted in figure $8 \mathrm{~d}$ ) is roughly represented by a steep increase of $W_{77}$ from $M_{V} \sim+3$ to $\sim 0$, followed by only gradual increase from $M_{V} \sim 0$ to $\sim-4$.

Accordingly, regarding the question about the applicability limit of published $W_{77}$ vs. $M_{V}$ formulas, which was raised in section 1 and motivated this study, our answer is as follows:

- (1) These analytical relations may be safely applied to intrinsically bright supergiants $\left(-5 \gtrsim M_{V} \gtrsim-10\right)$, which have low gravities of $\log g \lesssim 2.5$ in any case. However, highprecison would not be expected in empirically estimating $M_{V}$ from $W_{77}$, because appreciable dispersion amounting to $\lesssim 2 \mathrm{mag}$ in $M_{V}$ is observed in those proposed formulas (cf. gray lines in figure $8 \mathrm{~d}$ ). Moreover, our results (larger open circles in figure 8d) show some systematic difference (our $M_{V}$ values show a rather large scatter but tend to be dimmer by $\sim 1-2 \mathrm{mag}$ on the average) as compared to these published relations. This manifestly reflects the general difficulty of $M_{V}$ determination for such bright supergiants suffering large unertainties in $A_{V}$ as well as in $\pi$ because they are distantly located in the Galactic plane (note that the error in $M_{V}$ begins to prominently grow from several tenths mag at $M_{V} \sim-2$ to a considerable level of $\sim 1 \mathrm{mag}$ or even larger at $M_{V} \sim-5$; see figure $7 \mathrm{c}$ ). - (2) As to giants or bright giants of $0 \gtrsim M_{V} \gtrsim-5$, the situation is complicated because $\log g \lesssim 2.5$ group and $\log g \gtrsim 2.5$ group show markedly different trends. While the simple conventional relations (such as that proposed by Arellano Ferro et al. 2003, which well fits our results of small open circles in figure 8d) may still be usable for the former (typically F-G supergiants), they are no more valid for the latter (typically $\mathrm{A}$ giants). If $W_{77}$ values of these mixed samples altogether are to be explained by analytical relations, one would have to invoke complex formulas depending not only on $M_{V}$ but also on other stellar parameters.

- (3) In short, regarding the luminosity effect of $\mathrm{O} \mathrm{I}$ 7771-5 triplet, application (or devising) of useful analytical $W_{77}$ vs. $M_{V}$ formulas had better be confined to only bright supergiants of $-5 \gtrsim M_{V} \gtrsim-10$. We should bear in mind, however, that such relations are quantitatively of limited accuracy, because of the enormous difficulty in precisely calibrating $M_{V}$ of such generally distant stars suffering large interstellar extinction.

\subsection{Comparison with theoretical calculations}

Now that the observed trend of O I 7771-5 line strength in terms of stellar parameters has been elucidated, it is meaningful to check how this is compared with theoretically computed values of $W_{77}$. In our line-formation calculation, the non-LTE effect was taken into account based on plane-parallel model atmospheres, in which microturbulence was assumed to be depth-independent. We consider this treatment/assumption is practically sufficient (even if not complete), since the behaviors of non-LTE effect and (especially) microturbulence, both of which tend to increase toward lower-density atmospheres of higherluminosity stars, should be the main factors that dominate this luminosity effect.

Although Przybilla et al. (2000) suggested that nonclassical nature of stellar atmospheres (spherical extension effect, outflow velocity field) may be involved with the considerable intensification of O I 7771-5 lines in A-type supergiants, their argument does not seem to be based on a firm evidence. Regarding normal high-mass supergiants with static atmospheres, the sphericity effect is not significant and application of plane-parallel model atmospheres is not bad in the practical sense, since the thickness of atmosphere $(d)$ is still considerably smaller than the radius $(R) .{ }^{10}$ Admittedly, the situation must be different for the case of expanding atmospheres with significant mass loss. However, it seems unlikely that such an unusual phenomenon is commonly relevant for $\mathrm{A}-\mathrm{F}-\mathrm{G}$ giants and supergiants under study.

We computed $W_{77}$ (non-LTE and LTE, solar oxygen abundance, corresponding to $3 \xi$ values of 2,5 , and $10 \mathrm{~km} \mathrm{~s}^{-1}$ ) on a grid of models resulting from combinations of 11 values of $T_{\text {eff }}(5000,5500, \cdots 10000 \mathrm{~K})$ and 6 values of $\log g(1.5,2.0, \cdots 4.0)$, as done for computing the grid of non-LTE departure coefficients (section 4). Figures $9 \mathrm{a}$ and $9 \mathrm{~b}$ show the run of $W_{77}^{\mathrm{N}}$ as well as $W_{77}^{\mathrm{L}}$ with $T_{\text {eff }}$ (for $\log g=2.5$ case) and that with $\log g$ (for $T_{\text {eff }}$ $=7500 \mathrm{~K}$ case), respectively. It can be observed that $W_{77}^{\mathrm{N}}$ increases as $T_{\text {eff }}$ becomes higher up to $\sim 8000 \mathrm{~K}$ where it attains a broad peak. We also see that the line is conspicuously intensified by the non-LTE effect and by increasing $\xi$, and the non-LTE strengthening grows as $\log g$ is decreased. (especially for A-type stars).

The theoretical $W_{77}^{\mathrm{N}}$ vs. $M_{V}$ plots for three different $T_{\text {eff }}$ groups are depicted in figures $9 \mathrm{c}, 9 \mathrm{~d}$, ad $9 \mathrm{e}$, where the empirical trends are also shown by solid lines. We can recognize from these figures that these relations computed with given (constant) $\xi$ values generally fail to explain the observed data; i.e., the growth of observed $W_{77}$ with a decrease in $M_{V}$ (increasing luminosity) is much steeper than

10 Since $R$ and $d$ can be expressed as $R \propto T_{\text {eff }}^{-2} L^{1 / 2}$ and $d \propto$ $g^{-1} \propto R^{2} M^{-1} \propto T_{\text {eff }}^{-4} L M^{-1}$, we have $d / R \propto T_{\text {eff }}^{-2} L^{1 / 2} M^{-1}$. Accordingly, as $d / R$ is inversely proportional to $M$, the sphericity effect is not important for ordinary supergiants of large $M$, since extended $d$ due to low gravity tends to be compensated by expanded $R$. We should note, however, that this effect can be significant for low-mass and low-gravity stars (classified superficially as supergiants), such as the case of post-AGB stars. 
the theoretical expectation. This means that an increase of $\xi$ as a star becomes brighter is essential to explain the observed $M_{V}$-dependence of $W_{77}$. We should note, however, that $\log g \gtrsim 2.5$ stars at $0 \gtrsim M_{V} \gtrsim-4$ may belong to an exceptional case, since the observed gradual trend (red line) almost match the theoretical gradient. This indicates that $\xi$ values of these stars may not show any significant dependence on $M_{V}$ (see also subsection 7.4).

\subsection{Trend of microturbulence}

Finally, we discuss how the microturbulence depends on stellar parameters based on the two kinds of $\xi$ results derived in section $5\left(\xi_{\mathrm{p}}\right)$ and section $6\left(\xi_{\mathrm{a}}\right)$. These $\left(\xi_{\mathrm{p}}\right.$, $\left.\xi_{\mathrm{a}}\right)$ are plotted against $T_{\mathrm{eff}}, \log g$, and $v_{\mathrm{M}}$ in figures $10 \mathrm{a}, \mathrm{a}^{\prime}$, $10 \mathrm{~b}, \mathrm{~b}^{\prime}$, and $10 \mathrm{c}, \mathrm{c}^{\prime}$, respectively. The correlation of $\xi_{\mathrm{p}}$ and $\xi_{\mathrm{a}}$ is shown in figure $10 \mathrm{~d}$.

It can be seen from figures $10 \mathrm{~d}$ that $\xi_{\mathrm{p}}$ and $\xi_{\mathrm{a}}$ are more or less consistent with each other, despite that these two were derived by different approaches. Actually, the distribution of $\xi_{\mathrm{p}}$ in figure 10a,b,c (left panels) and that of figure $10 \mathrm{a}^{\prime}, \mathrm{b}^{\prime}, \mathrm{c}^{\prime}$ (right panels) appear almost similar. A notable exception is that $\xi_{\mathrm{p}}$ tends to be appreciably larger than $\xi_{\mathrm{a}}$ for four A-type supergiants (large open circles in figure 10d) where $\mathrm{O}$ I $7771-5$ feature is very strong $\left(W_{77}>1 \AA\right)$. We suspect that this difference is related to the depth-dependence of $\xi$, because this parameter is likely to increase with atmospheric height in supergiants (see, Appendix B of Takeda \& Takada-Hidai 1994). That is, since $\xi_{\mathrm{p}}$ is mainly determined by core profile of strong feature where the formation depth is higher, it tends to reflect the condition of upper atmosphere (where $\xi$ is larger than the deeper layer), which eventually leads to $\xi_{\mathrm{p}}>\xi_{\mathrm{a}}$. Actually, a similar phenomenon was previously observed by Takeda et al. (1996; cf. Sect. 5.1.1 therein) for the Sun and Procyon (though the inequality relation was reversed in that case of dwarfs, indicating a decrease of $\xi$ with height).

We can see from figure $10 \mathrm{a}, \mathrm{a}^{\prime}$ that the $\xi$ vs. $T_{\text {eff }}$ relations for A dwarfs and FGK dwarfs (solid lines) almost coincide with the lower envelope of the $\xi$ distribution derived for our program stars. Accordingly, a growing of $\xi$ is a natural tendency of evolved A-G stars, which generally occurs as a star leaves off the main sequence.

Figure $10 \mathrm{~b}, \mathrm{~b}^{\prime}$ shows how $\xi$ depends on $\log g$, where we can observe that $\xi$ roughly grows with a decrease of $\log g$. This suggests that turbulence tends to be enhanced as the atmospheric density is lowered, which is intuitively reasonable. ${ }^{11}$ However, this trend is not simply monotonic but rather intricate. For example, $\xi$ values of A supergiants (large open circles) are distinctly larger than those of $\mathrm{F}-\mathrm{G}$ supergiants (small open circles) at the same $\log g \sim 2$. We also see that, for stars of $3.0 \gtrsim \log g \gtrsim 2.5$ (filled circles), $\xi\left(\sim 3-4 \mathrm{~km} \mathrm{~s}^{-1}\right)$ is rather inert to a change of $\log g$. Since stars of this $\log g$ range almost correspond to a $M_{V}$ range between $\sim 0$ and $\sim-5$ according to equation $(1)$, this indicates the existence of stagnant trend in $\xi$ already

11 This tendency is seen also in late $\mathrm{G}$ through early $\mathrm{K}$ giants (evolved stars with lower $T_{\text {eff }}$ ), as shown in Fig. 1c of Takeda, Sato, and Murata (2008b). suggested in subsection 7.3. Further noteworthy is the sign of $\xi$ hump (up to $\lesssim 10 \mathrm{~km} \mathrm{~s}^{-1}$ ) for stars of $\log g \sim 3$ (these have $T_{\text {eff }} \sim 6000 \mathrm{~K}$ ), which is not easy to interpret.

Regarding the $\xi$ vs. $v_{\mathrm{M}}$ relation shown in figure $10 \mathrm{c}, \mathrm{c}^{\prime}$, what we can state is almost similar to what we already discussed about the $v_{\mathrm{M}}$-dependence of $W_{77}$ in subsection 7.2. The steep increase of $\xi$ with $v_{\mathrm{M}}$ at $0 \mathrm{~km} \mathrm{~s}^{-1} \lesssim$ $v_{\mathrm{M}} \lesssim 40 \mathrm{~km} \mathrm{~s}^{-1}$ for $\log g<2.5$ stars must be due to the close correlation between macroturbulence and microturbulence, since macroturbulence significantly contributes to (or dominates) $v_{\mathrm{M}}$ in this range of comparatively small $v_{\mathrm{M}}$. For large $v_{\mathrm{M}}$ region (more than several tens $\mathrm{km} \mathrm{s}^{-1}$ ) where $v_{\mathrm{M}} \simeq v_{\mathrm{e}} \sin i$ almost holds, we can not observe any meaningful dependence of $\xi$ upon $v_{\mathrm{M}}$. Accordingly, microturbulence is not likely to be explicitly affected by stellar rotation.

\section{Conclusion}

Although the strength of $\mathrm{O}$ I $7771-5$ feature is known to show a luminosity effect and may be usable for empirical evaluation of absolute magnitude, our understanding on its behavior is still insufficient. Especially, the validity and applicability limit of various analytical relations proposed so far (which are not necessarily consistent with each other) has yet to be clarified, for which comprehensive study on the parameter dependence of $W_{77}$ for evolved stars in general would be needed.

With an aim to shed light on these points, we carried out an extensive non-LTE spectrum-fitting analysis of O I 7771-5 lines for unbiased sample of 75 evolved A-, F,- and G-type stars of various luminosity classes (subgiants, giants, and supergiants) including rapid rotators, from which $W_{77}$ was derived for each star. Besides, as a by-product of analysis, we determined the microturbulence (which plays a significant role in controlling $W_{77}$ ) by two different approaches (profile-based method and abundance-based method) for each star, because its behavior of evolved stars across the HR diagram is not yet well understood.

We confirmed that the resulting $W_{77}$ values of the program stars tend to increase as $M_{V}$ becomes more luminous. However, the behavior of $W_{77}$ for the whole sample is too complicated to be described by a simple relation. Specifically, distinctly different trends of $W_{77}$ were found for the lower-gravity $(\log g \lesssim 2.5)$ group and the highergravity $(\log g \gtrsim 2.5)$ group; and the simple $M_{V}$ vs. $W_{77}$ formulas proposed by past studies are applicable only to the former group, but not to the latter group which shows a totally different tendency. Since these two groups overlap at $0 \gtrsim M_{V} \gtrsim-5$ (i.e., $\mathrm{F}-\mathrm{G}$ supergiants of the former group and A-type giants of the latter group), special care should be taken in using $W_{77}$ of stars in this $M_{V}$ range., It is thus recommended to confine only to supergiants of $-5 \gtrsim M_{V} \gtrsim-10$, if one wants to safely make use of the luminosity effect of $\mathrm{O}$ I $7771-5$ lines.

Concerning our question raised in section 1 (see the second paragraph therein), which motivated this investigation, our conclusion is that the relation between $M_{V}$ 
and $W_{77}$ over a wide $M_{V}$ range (covering giants and supergiants from $\sim 0 \mathrm{mag}$ to $\sim-9 \mathrm{mag}$ ) can not be represented by such a simple analytical formula as derived by Arellano Ferro et al. (2003). Presumably, their sample stars (which they used for calibration to derive the formula) were not so sufficiently diversified as to detect the dispersion of $W_{77}$ for giants of $M_{V} \gtrsim-5$.

Regarding the behavior of microturbulence, it roughly shows an increasing tendency with a decrease in surface gravity, which we believe to be the primary cause of the luminosity-dependence of $W_{77}$. However, the trend is not monotonic but rather intricate depending on the stellar type and evolutionary stage (e.g., hump around $\log g \sim 3$, stagnation at $\log g \sim 3-3.5$, or discontinuously large increase up to $\gtrsim 10 \mathrm{~km} \mathrm{~s}^{-1}$ for luminous A-type supergiants). These results may be used as observational constraints for investigating the nature of velocity fields in the atmosphere of evolved $\mathrm{A}-\mathrm{F}-\mathrm{G}$ stars.

The first author (Y. T.) heartily thanks Dr. J. Hakkila for informing how to get his EXTINCT code. This research has made use of the SIMBAD database, operated at CDS, Strasbourg, France. Data reduction was in part carried out by using the common-use data analysis computer system at the Astronomy Data Center (ADC) of the National Astronomical Observatory of Japan.

\section{References}

Alonso, A., Arribas, S., \& Martínez-Roger, C. 1999, A\&AS, 140, 261 [Erratum: A\&A, 376, 1039 (2001)]

Arellano Ferro, A., Giridhar, S., Chavez, M., \& Parrao, L. 1989, A\&A, 214, 123

Arellano Ferro, A., Giridhar, S., \& Goswami, A. 1991, MNRAS, 250, 1

Arellano Ferro, A., Giridhar, S., \& Rojo Arellano, E. 2003, RMA\&A, 39, 3

Arellano Ferro, A., \& Mendoza, E. E. 1993, AJ, 106, 2516

Baker, P. W. 1974, PASP, 86, 33

Cayrel, R. 1988, in The Impact of Very High S/N Spectroscopy on Stellar Physics, Proc. IAU Symp. 132, eds. G. Cayrel de Strobel \& M. Spite (IAU), p. 345

Dambis, A. K. 2013, in Advancing the Physics of Cosmic Distances, Proc. IAU Symp. 289, ed. R. de Grijs (IAU), 379

ESA 1997, The Hipparcos and Tycho Catalogues, ESA SP1200 , available from NASA-ADC or CDS in a machinereadable form (file name: hip_main.dat)

Faraggiana, R., Gerbaldi, M., van't Veer, C., \& Floquet, M. 1988, A\&A, 201, 259

Flower, P. J. 1996, ApJ, 469, 355

Gray, D. F. 1978, Sol. Phys., 59, 193

Gray, D. F. 2005, The Observation and Analysis of Stellar Photospheres, 3rd ed. (Cambridge: Cambridge University Press)

Hakkila, J., Myers, J. M., Stidham, B. J., \& Hartmann, D. H. 1997, AJ, 114, 2043

Hopkinson, G. R., \& Humrich, A. 1981, MNRAS, 195, 661

Kameswara Rao, N., \& Mallik, S. G. V. 1978, MNRAS, 183, 211

Keenan, P. C., \& Hynek, J. A. 1950, ApJ, 111, 1
Kurucz, R. L. 1993, Kurucz CD-ROM, No. 13 (HarvardSmithsonian Center for Astrophysics)

Kurucz, R. L., \& Bell, B. 1995, Kurucz CD-ROM, No. 23 (Harvard-Smithsonian Center for Astrophysics)

Kovtyukh, V. V., Gorlova, N. I., \& Belik, S. I. 2012, MNRAS, 423, 3268

Lee, B.-C., Galazutdinov, G. A., Han, I., Kim, K.-M., Yushchenko, A. V., Kim, J., Tsymbal, V., \& Park, M.-G. 2006, PASP, 118, 636

Lejeune, T., \& Schaerer, D. 2001, A\&A, 366, 538

Mendoza, E. E., \& Arellano Ferro, A. 1993, AJ, 106, 2524

Merrill, P. W. 1925, PASP, 37, 272

Osmer, P. S. 1972, ApJS, 24, 247

Przybilla, N., Butler, K., Becker, S. R., Kudritzki, R. P., \& Venn, K. A. 2000, A\&A, 359, 1085

Slowik, D. J., \& Peterson, D. M. 1993, AJ, 105, 1967

Slowik, D. J., \& Peterson, D. M. 1995, AJ, 109, 2193

Sorvari, J. M. 1974, AJ, 79, 1416

Takeda, Y. 1992, PASJ, 44, 309

Takeda, Y. 1995, PASJ, 47, 287

Takeda, Y. 2003, A\&A, 402, 343

Takeda, Y., Han, I., Kang, D.-I., Lee, B.-C., \& Kim, K.-M. 2008a, JKAS, 41, 83

Takeda, Y., Hashimoto, O., \& Honda, S. 2017, PASJ, 69, 1

Takeda, \& Honda, S. 2005, PASJ, 57, 65

Takeda, Y., Honda, S., Ohnishi, T., Ohkubo, M., Hirata, R., \& Sadakane, K. 2013, PASJ, 65, 53

Takeda, Y., Kato, K., Watanabe, Y., \& Sadakane, K. 1996, PASJ, 48, 511

Takeda, Y., \& Sadakane, K. 1997, PASJ, 49, 367

Takeda, Y., Sato, B., \& Murata, D. 2008b, PASJ, 60, 781

Takeda, Y., \& Takada-Hidai, M. 1994, PASJ, 46, 395

van Leeuwen, F. 2007, Hipparcos, the New Reduction of the Raw Data (Berlin: Springer) 


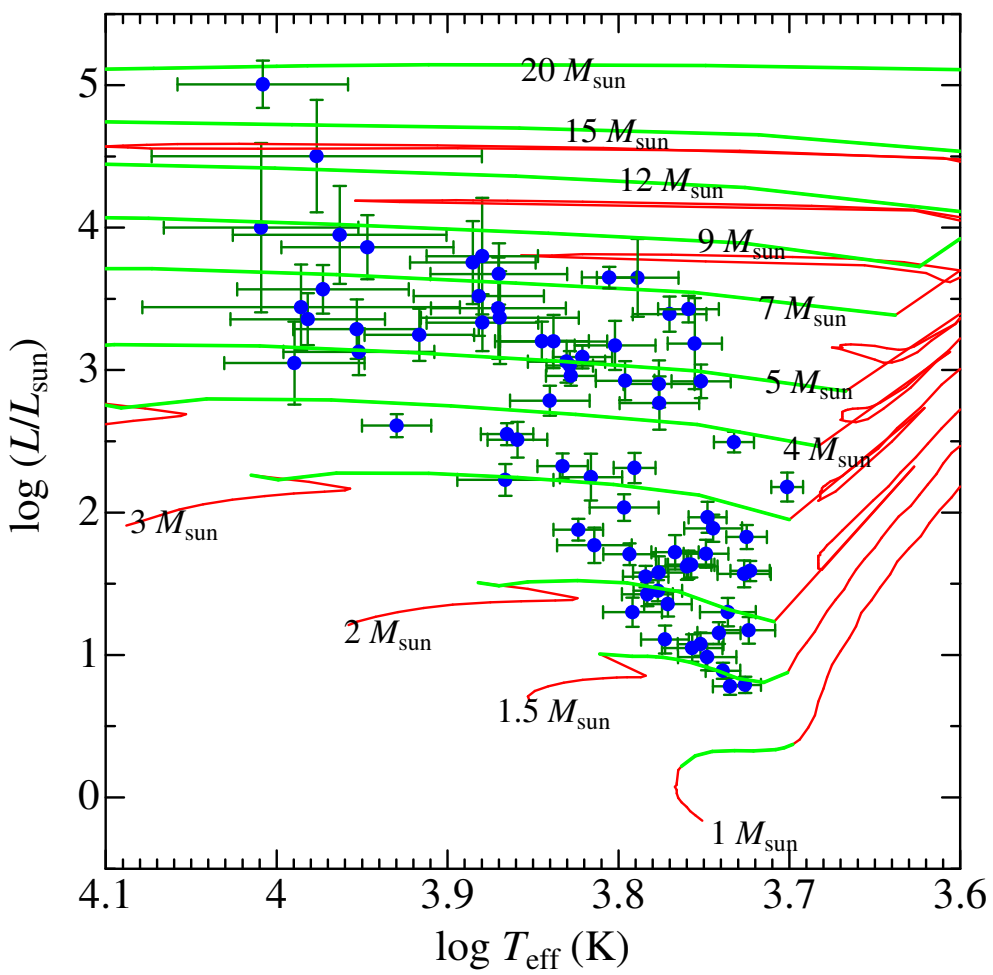

Fig. 1. Our 75 program stars plotted on the theoretical HR diagram $\left(\log \left(L / L_{\odot}\right)\right.$ vs. $\left.\log T_{\text {eff }}\right)$, where $T_{\text {eff }}$ was determined from the $B-V$ color (corrected for interstellar reddening) while $L$ was evaluated from visual magnitude (corrected for interstellar extinction), Hipparcos parallax, and bolometric correction. The error bars in $T_{\text {eff }}$ are due to ambiguities of interstellar reddening, while those in $L$ are estimated by combining the uncertainties of interstellar extinction and of Hipparcos parallax. Theoretical solar-metallicity tracks computed by Lejeune and Schaerer (2001) are also depicted for 11 different masses $\left(1,1.5,2,3,4,5,7,9,12,15\right.$, and $\left.20 M_{\odot}\right)$ for comparison. The nearly horizontal parts of the tracks (corresponding to the shell-hydrogen burning phase) colored in light-green were used to estimate $M$ for given $L$ and $T_{\text {eff }}$ by interpolation. 

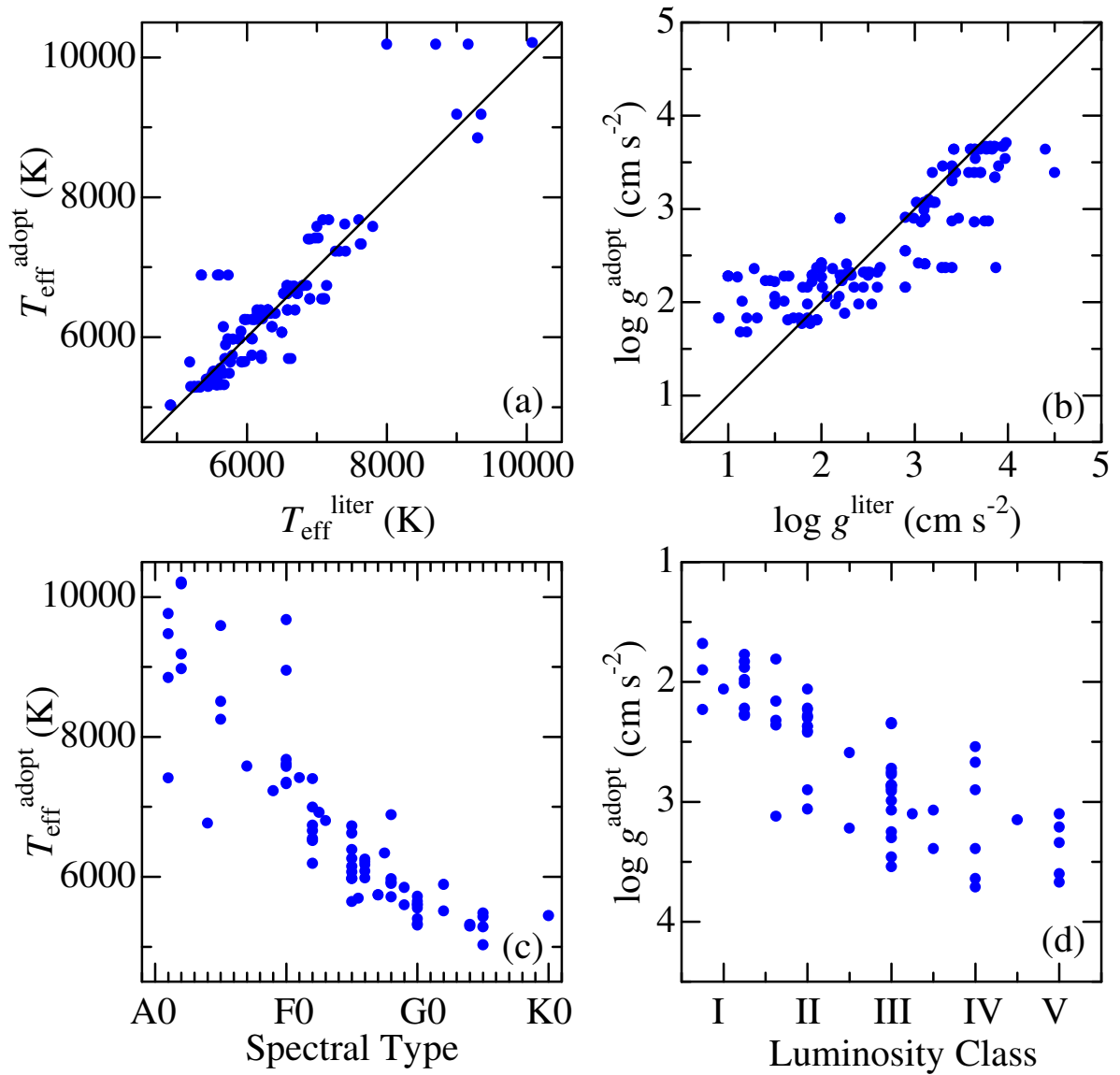

Fig. 2. Panels (a) and (b) show the comparison of $T_{\text {eff }}$ and $\log g$ adopted in this study with those of literature data taken from the simbad database (available for $\sim 40$ stars, though the number of the plotted points is larger because several published data tend to be attached for each star). In panels (c) and (d) are depicted the correlations of $T_{\text {eff }}$ vs. spectral type and log $g$ vs. luminosity class, respectively, where subtypes were tentatively digitized (such as like Ia $\rightarrow 0.75$ and $\mathrm{Ib} \rightarrow 1.25$ ). 

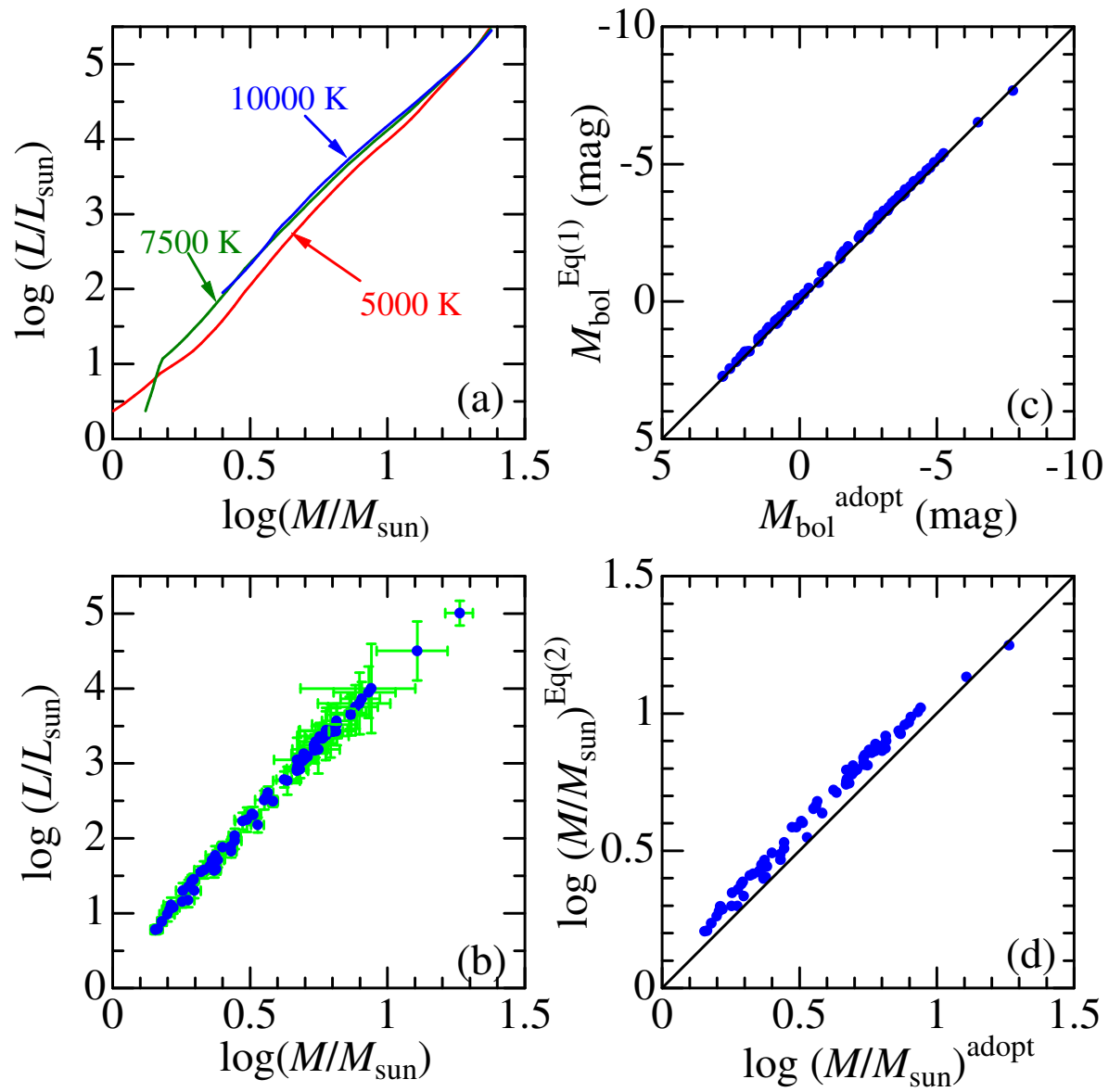

Fig. 3. (a) $L$ vs. $M$ relation (for given $T_{\text {eff }}$ ) derived from the near-horizontal parts (colored in light-green in figure 1 ) of the evolutionary tracks. Depicted here are the cases of $T_{\text {eff }}=5000,7500$, and $10000 \mathrm{~K}$, where we can see that almost the same relation holds irrespective of $T_{\text {eff }}$. (b) Mass $(M)$ values of the program stars (which were derived by using these $M=f\left(L, T_{\text {eff }}\right)$ relations by interpolation) plotted against luminosity $(L)$. The attached error bars are due to the ambiguities in $L$ (see the caption in figure 1 ). (c) Correlation of $M_{\mathrm{bol}}$ (absolute bolometric magnitude) values derived in this study with those computed by equation (1). (d) Correlation of $M$ (mass) values derived in this study with those computed by equation (2). 


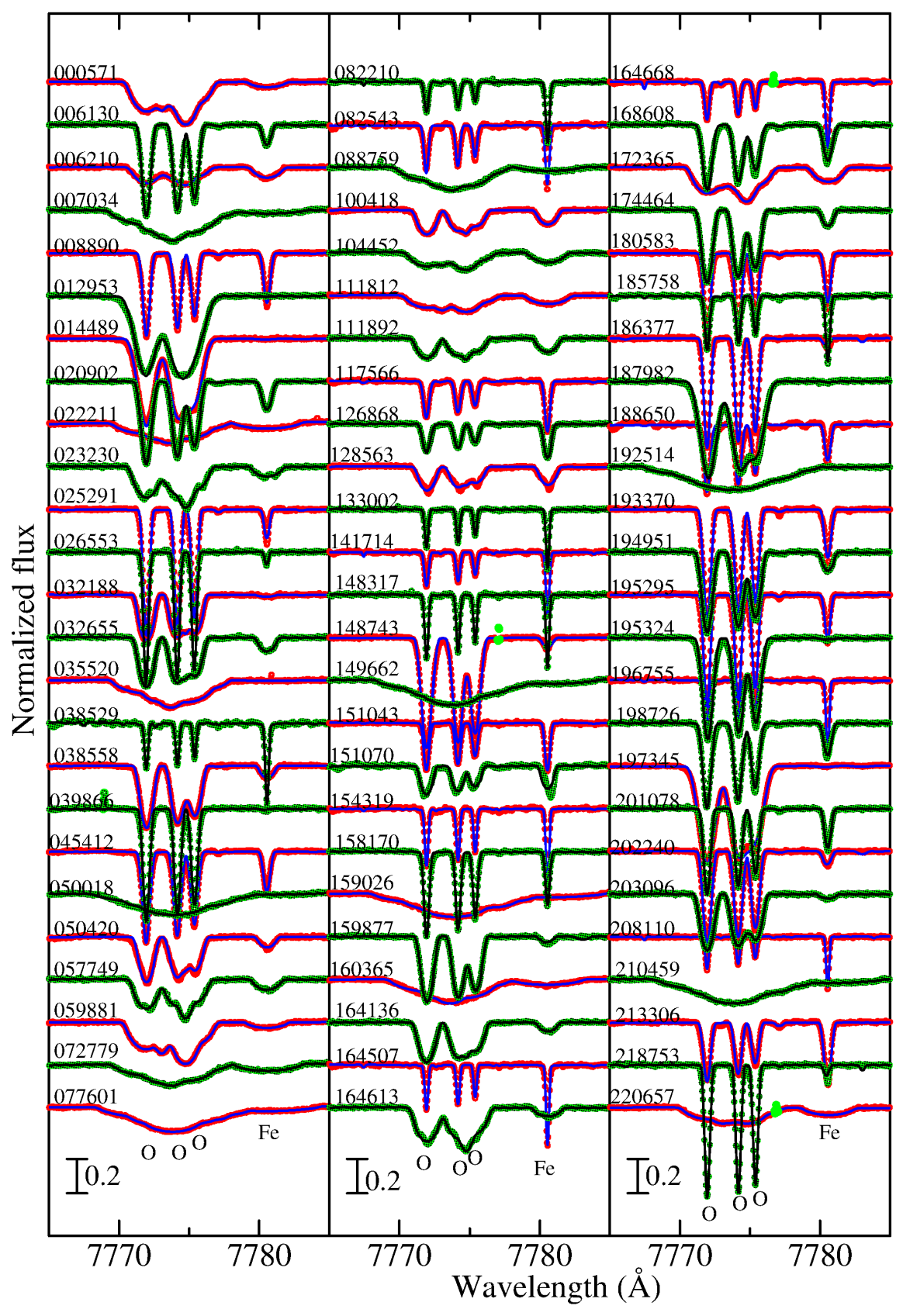

Fig. 4. Synthetic spectrum fitting at the 7765-7785 $\AA$ region. The best-fit theoretical spectra are shown by solid lines and the observed data are plotted by symbols (while those masked/disregarded in the fitting are highlighted in light-green). In each panel, the spectra are arranged in the order of star's HD number (indicated in the figure) as in table 2, and an offset of 0.2 is applied to each spectrum relative to the adjacent one. The wavelength scale is adjusted to the laboratory frame by correcting the radial-velocity shift. 


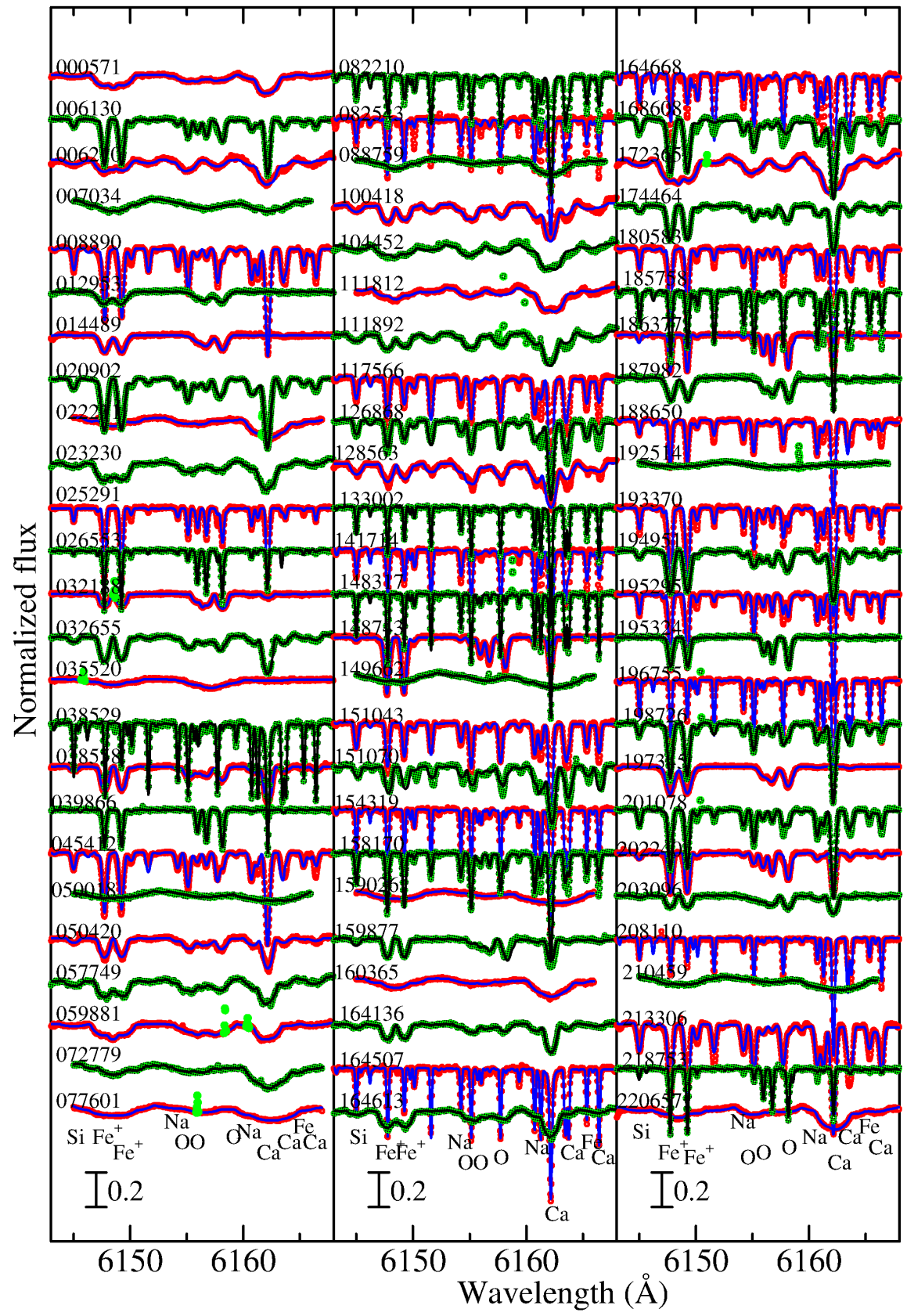

Fig. 5. Synthetic spectrum fitting at the 6143-6168 $\AA$ region. Otherwise, the same as in figure 4 . 


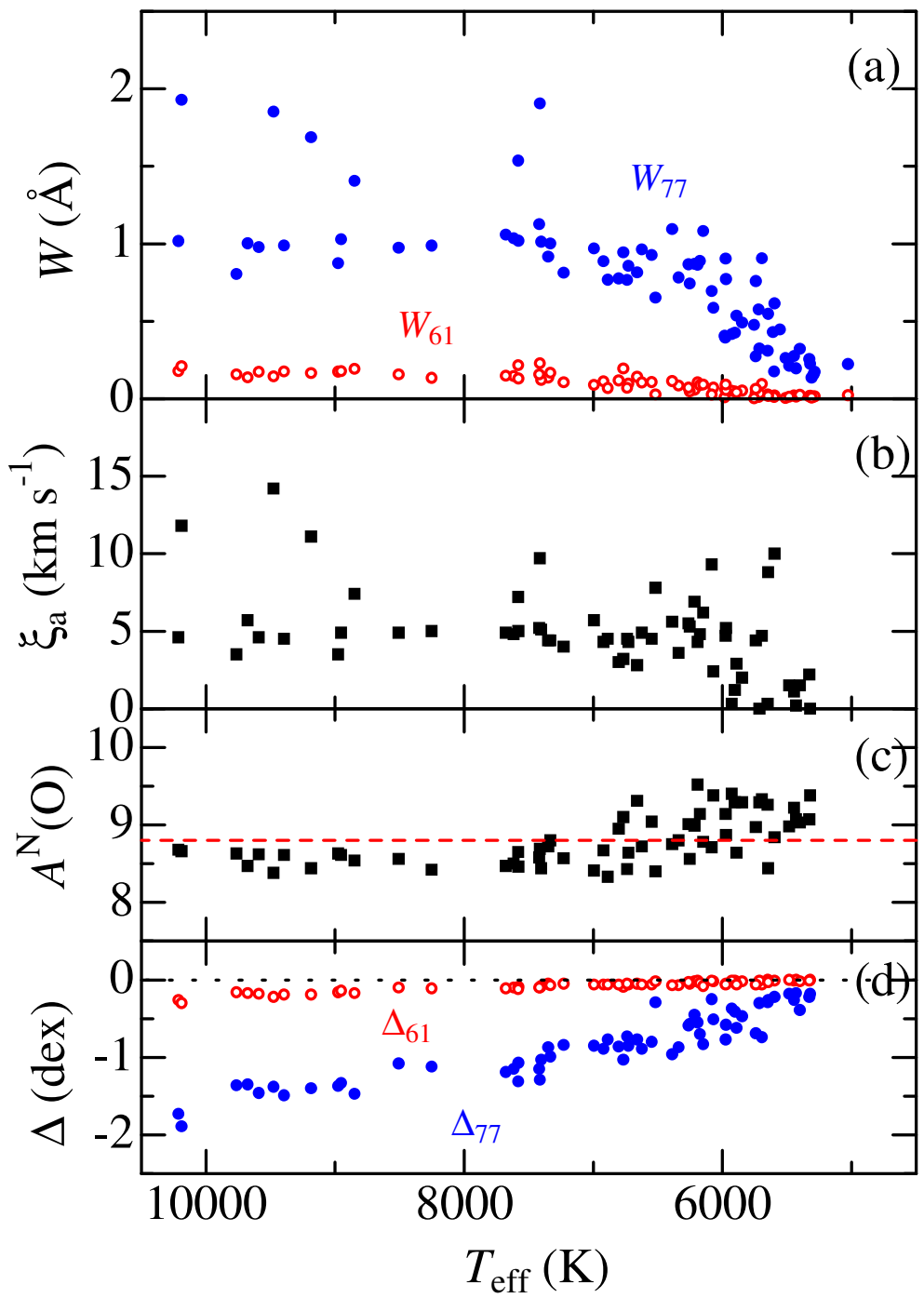

Fig. 6. Oxygen abundances and related quantities for O I 7771-5 (suffix "77") and O I 6155-8 (suffix "61") lines, plotted against

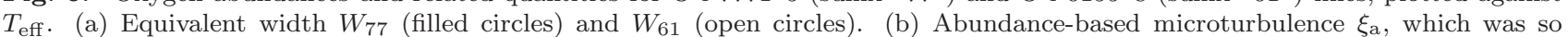
determined that two non-LTE $\mathrm{O}$ abundances $\left(A_{77}^{\mathrm{N}}\right.$ and $\left.A_{61}^{\mathrm{N}}\right)$ equal to each other. (c) Non-LTE oxygen abundance $A^{\mathrm{N}}(\mathrm{O})\left[=A_{77}^{\mathrm{N}}(\mathrm{O})\right.$ $\left.=A_{61}^{\mathrm{N}}(\mathrm{O})\right]$ corresponding to $\xi_{\mathrm{a}}$. (d) Non-LTE correction $\Delta_{77}$ (filled circles) and $\Delta_{61}$ (open circles), 

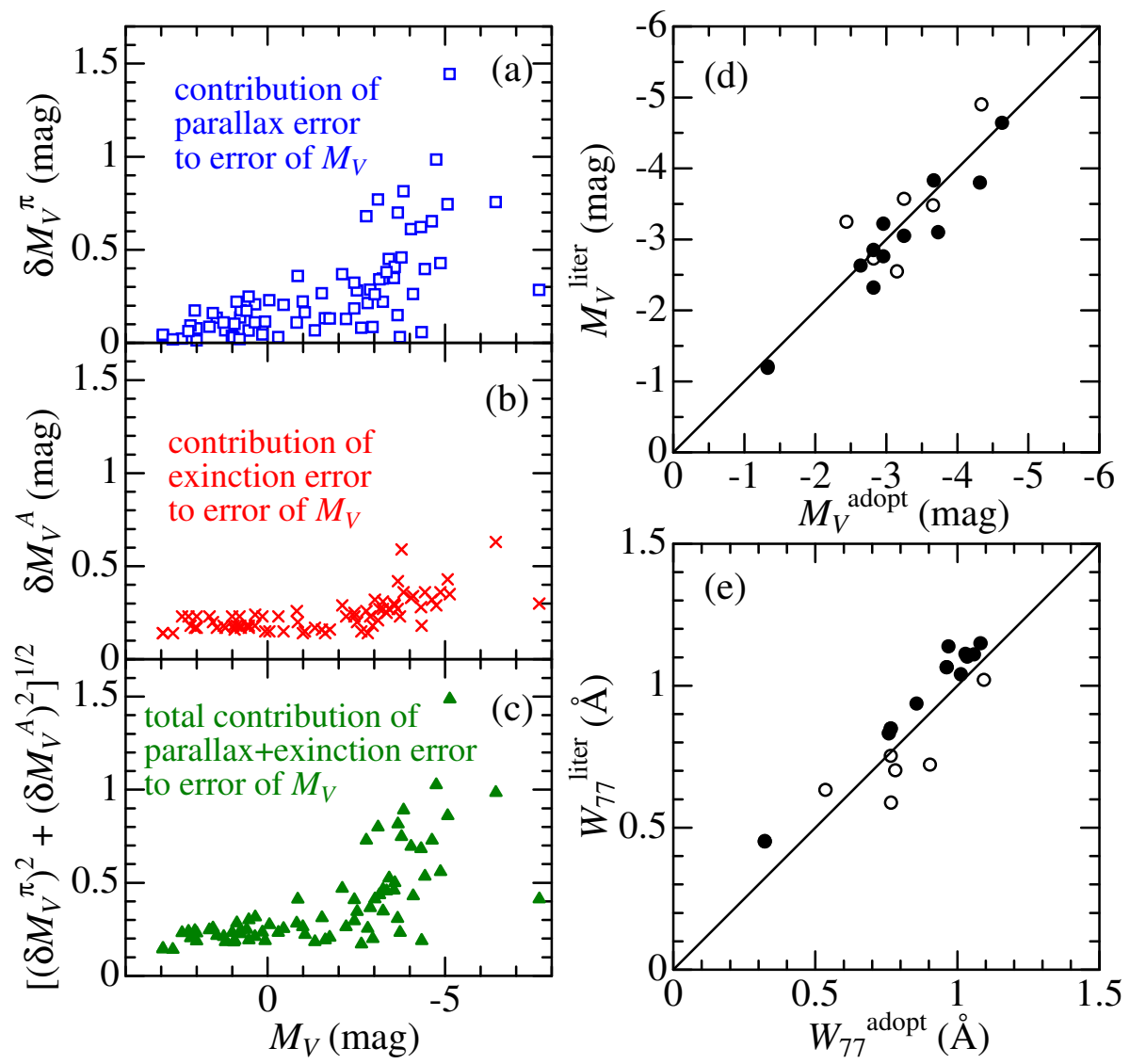

Fig. 7. The left-hand panels show how the errors in parallax or interstellar extinction contribute to errors in the absolute magnitude. (a) Error component $\left(\delta M_{V}^{\pi}\right)$ of $M_{V}$ due to error $\left(\sigma_{\pi}\right)$ in parallax $(\pi)$, which is defined as $\delta M_{V}^{\pi} \simeq 5 \log \left(1+\sigma_{\pi} / \pi\right)$, plotted against $M_{V}$. (b) Error component $\left(\delta M_{V}^{A}\right)$ of $M_{V}$ due to error in interstellar extinction $\left(A_{V}\right)$, plotted against $M_{V}$. (c) Total error of $M_{V}$, which is defined as the root-sum-square of $\delta M_{V}^{\pi}$ and $\delta M_{V}^{A}$, ploted against $M_{V}$. In the right-hand panels are shown the comparison of our adopted values of $M_{V}$ (panel d) or $W_{77}$ (panel e) with those of Arellano Ferro et al. (2003) (6 stars in common: open symbols) and Kovtyukh et al. (2012) (11 stars in common: filed symbols) 

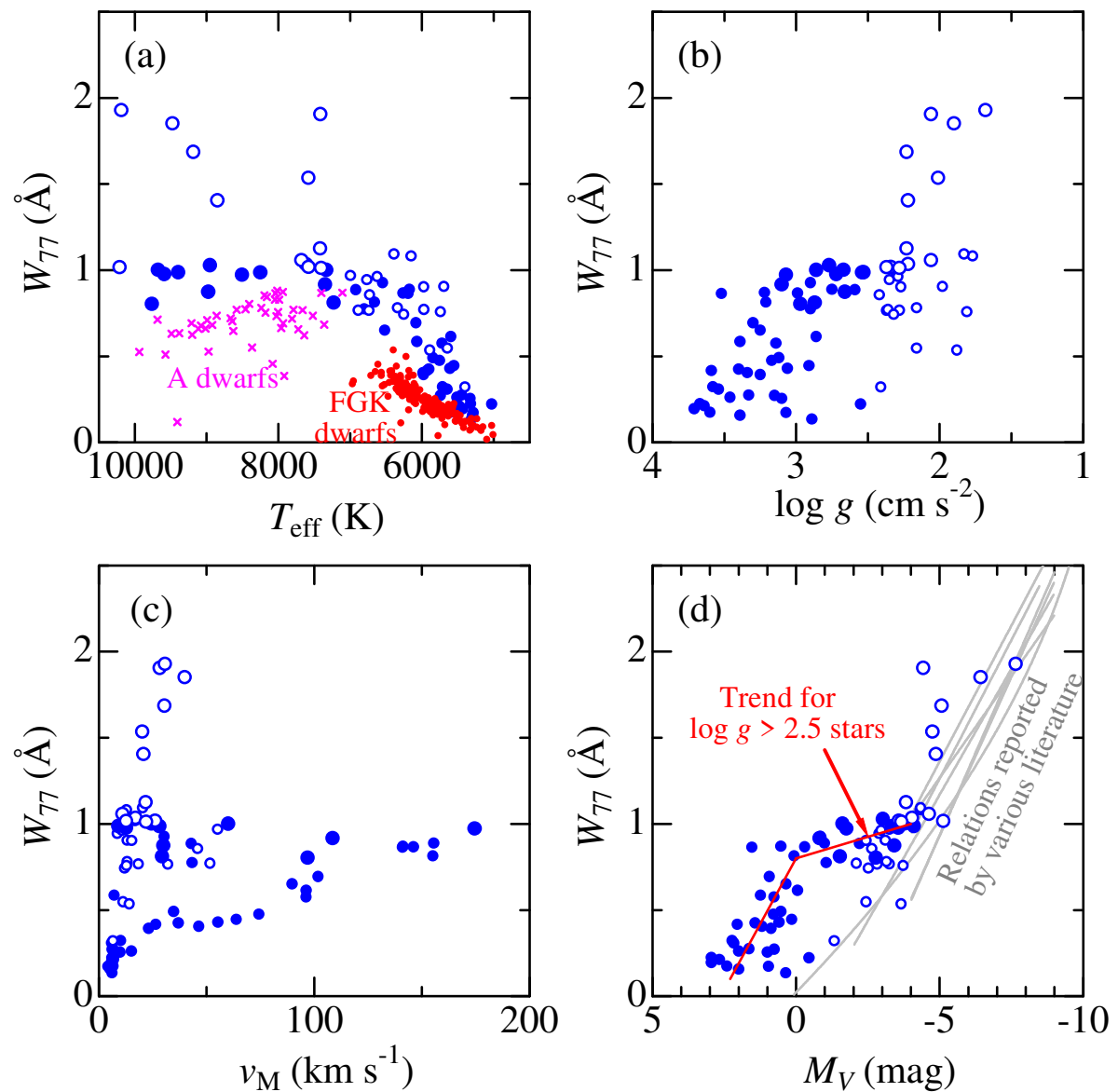

Fig. 8. The strength of the whole O I 7771-5 multiplet $\left(W_{77}\right)$ plotted against (a) effective temperature $\left(T_{\text {eff }}\right)$, (b) surface gravity $(\log g)$, (c) macrobroadening velocity $\left(v_{\mathrm{M}}\right)$, and $(\mathrm{d})$ absolute visual magnitude $\left(M_{V}\right)$. Open and filled circles denote stars of lower $\log g(<2.5)$ and higher $\log g(>2.5)$, respectively, while lower $T_{\text {eff }}(<7500 \mathrm{~K})$ and higher $T_{\text {eff }}(>7500 \mathrm{~K})$ stars are expressed in smaller and larger symbols. In panel (a), $W_{77}$ values of 160 FGK-type (red dots) and 46 A-type (pink crosses) main-sequence stars are shown for comparison. In panel (d), gray solid lines represent the seven published $W_{77}$ vs. $M_{V}$ relations given in table 1 (Ref. $1,2,3,7,11,12$, and 13 ), while the red solid line is the eye-estimated mean trend for $\log g>2.5$ stars (filled symbols). 

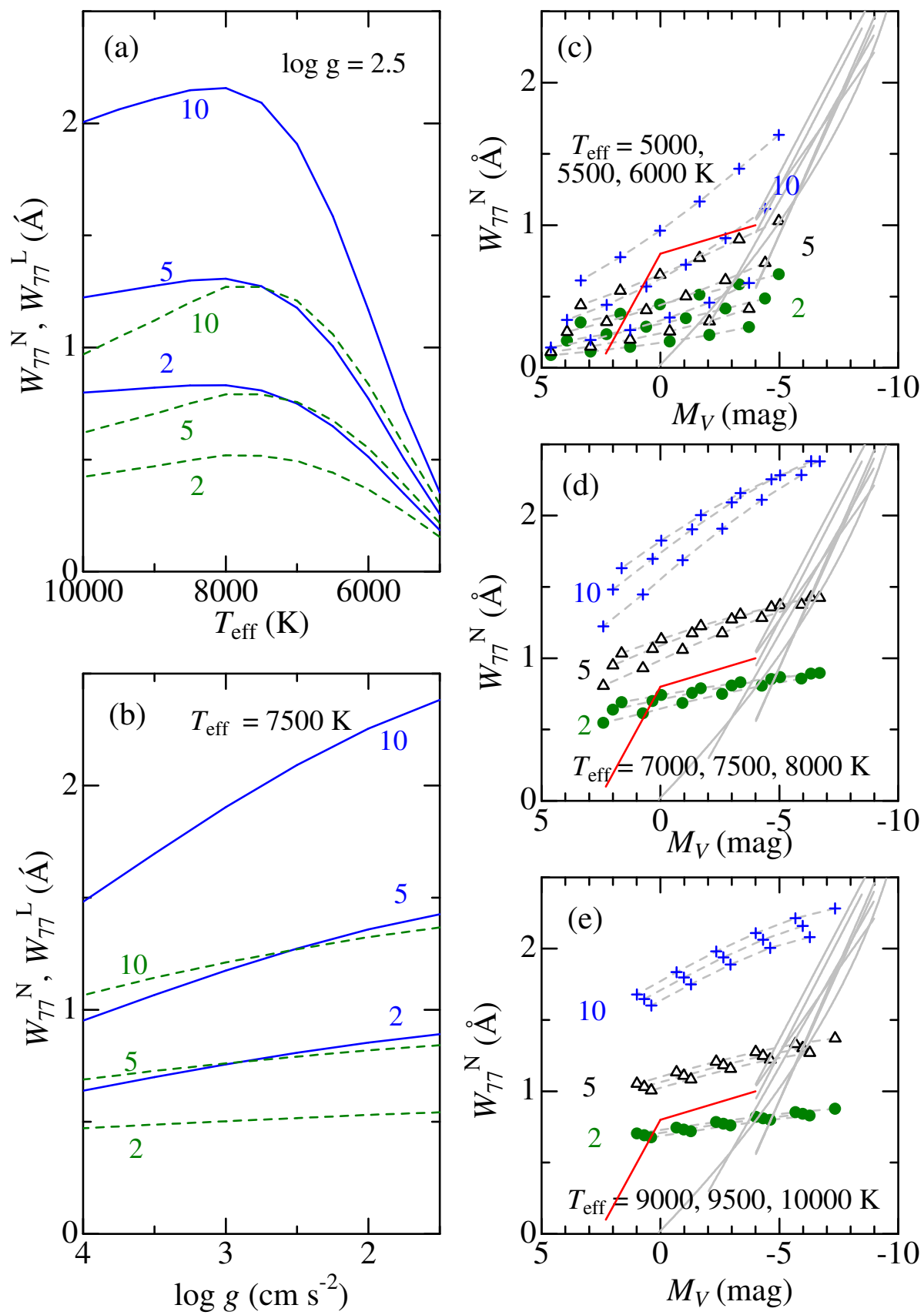

Fig. 9. Graphical display of how the theoretical $W_{77}$ (computed for the solar oxygen abundance) depends on the stellar parameters.

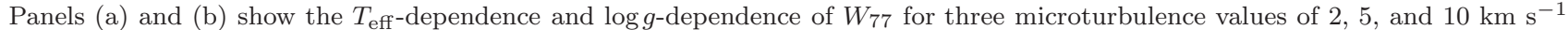
(indicated in the figure), where the solid and dashed lines represent non-LTE $\left(W_{77}^{\mathrm{N}}\right)$ and LTE $\left(W_{77}^{\mathrm{L}}\right)$ results, respectively. Panels (c), (d), and (e) illustrate the simulated dependence of $W_{77}^{\mathrm{N}}$ upon $M_{V}$ (evaluated by using equation (1) and Alonso et al.'s (1999)

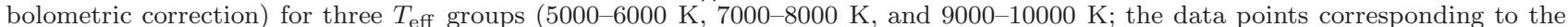
same $T_{\text {eff }}$ but different $\log g$ are connected by gray dashed lines), where the meanings of the solid lines are the same as in figure $8 \mathrm{~d}$. 

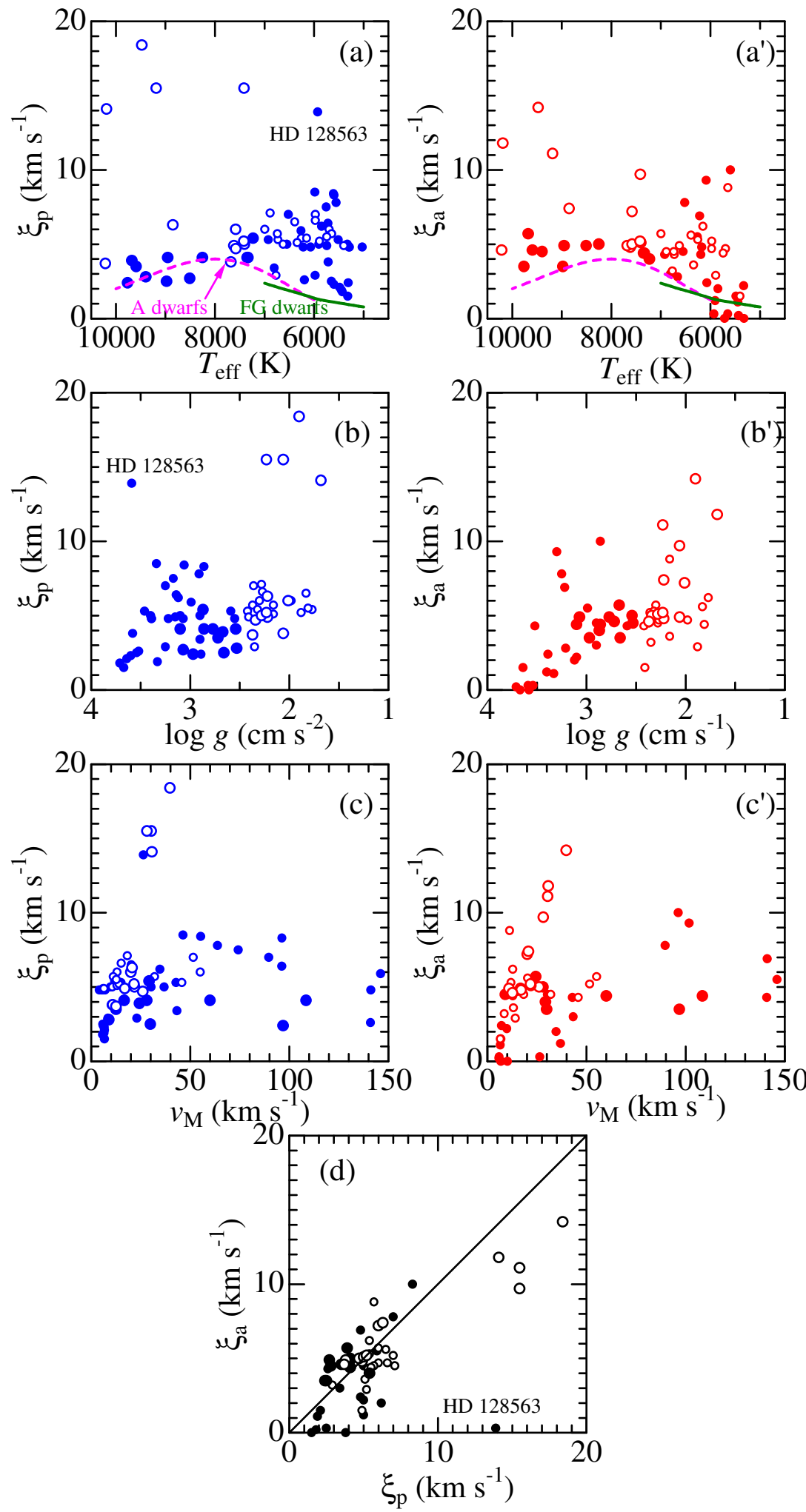

Fig. 10. Microturbulence values plotted against (a) $T_{\text {eff }}$, (b) $\log g$, and (c) $v_{\mathrm{M}}$, where the left panels (a, b, c) are for $\xi_{\mathrm{p}}$ (profile-based microturbulence) while the right panels $\left(\mathrm{a}^{\prime}, \mathrm{b}^{\prime}, \mathrm{c}^{\prime}\right)$ are for $\xi_{\mathrm{a}}$ (abundance-based microturbulence). The solid and dashed line in panels (a) and $\left(\mathrm{a}^{\prime}\right)$ are the mean trend of $\xi$ as a function of $T_{\text {eff }}$ for $\mathrm{F}-\mathrm{G}$ type main-sequence stars [Eq. (1) and (2) of Takeda et al. (2013) for the case of $\log g=4.0$ ] and A-type main-sequence stars [Eq. (1) of Takeda et al. (2008a)], respectively. The correlation between $\xi_{\mathrm{p}}$ and $\xi_{\mathrm{a}}$ is depicted in the bottom panel (d). The outlier result for HD 128563 is unreliable, since sharp components are weakly overlapped at the line positions. See the caption of figure 8 for the meanings of the symbols. 
Table 1. Representative work related to $\mathrm{O}$ I $7771-5$ line strength in $\mathrm{A}-\mathrm{F}-\mathrm{G}$ stars.

\begin{tabular}{|c|c|c|c|c|}
\hline No. & Reference & Star type* & Range of $M_{V}$ (mag) & $\bar{M}_{V}$ (mag) vs. $W_{77}(\AA)$ relation \\
\hline (1) & Osmer (1972) & F sg & from -4 to -9 & $M_{V}=-2.62 W_{77}-2.55$ \\
\hline$(2)$ & Baker (1974) & $\mathrm{F} \mathrm{sg}$ & from -4 to -9 & $M_{V}=-2.711 W_{77}-2.472$ \\
\hline (3) & Sorvari (1974) & F sg & from -2 to -9 & $M_{V}=-3.42 W_{77}-1.00$ \\
\hline (4) & Kameswara Rao \& Mallik (1978) & $\mathrm{F}-\mathrm{G} \operatorname{sg}$ & from -2 to -10 & $M_{V}=-10 E-1.79$ \\
\hline$(5)$ & Hopkinson \& Humrich (1981) & $\mathrm{A}-\mathrm{G} \operatorname{sg}$ & $\mathrm{N} / \mathrm{A}$ & $\mathrm{N} / \mathrm{A}$ \\
\hline (6) & Faraggiana et al. (1988) & B-F d-subg-g-sg & $\mathrm{N} / \mathrm{A}$ & $\mathrm{N} / \mathrm{A}$ \\
\hline$(7)$ & Arellano Ferro et al. (1989) & $\mathrm{F} \mathrm{sg}$ & from -4 to -9 & $M_{V}=-88.02 \Lambda(16)+187.83$ \\
\hline$(8)$ & Arellano Ferro et al. (1991) & $\mathrm{F}-\mathrm{G} \mathrm{g}-\mathrm{sg}$ & from +2 to -10 & $M_{V}=1.52-6.33 W_{77}+0.85 W_{77}^{2}-3.74(b-y)_{0}$ \\
\hline$(9)$ & Arellano Ferro \& Mendoza (1993) & $\mathrm{A}-\mathrm{G} \mathrm{g}-\mathrm{sg}$ & from +3 to -10 & $M_{V}=8.0-13.3 W_{77}+2.7 W_{77}^{2}-2.1(b-y)_{0}$ \\
\hline (10) & Mendoza \& Arellano Ferro (1993) & $\mathrm{A}-\mathrm{G} \mathrm{g}-\mathrm{sg}$ & from +3 to -8 & N/A \\
\hline (11) & Slowik \& Peterson (1993) & A-F sg & from -4 to -8.5 & $M_{V}=-1.07-3.11 W_{77}$ \\
\hline$(12)$ & Slowik \& Peterson (1995) & A-F sg & from -4 to -9 & $M_{V}=-0.68-3.17 W_{77}$ \\
\hline (13) & Arellano Ferro et al. (2003) & A-G g-sg, Cep & from +0.35 to -9.5 & $M_{V}=0.131-5.831 W_{77}+0.789 W_{77}^{2}$ \\
\hline (14) & Kovtyukh et al. (2012) & $\mathrm{F}-\mathrm{G}$ sg & from 0 to -9 & [cf. their Eq.(2)] \\
\hline (15) & Dambis (2013) & $\mathrm{F} \mathrm{sg}$ & from $M_{K}=-4.7$ to -9 & $M_{K}=-5.33-10.81 \log W_{77}$ \\
\hline
\end{tabular}

* Meaning of abbreviation: 'd' $\cdots$ dwarfs, 'subg' $\cdots$ subgiants, 'g' $\cdots$ giants, 'sg' $\cdots$ supergiants, and 'Cep' ... Cepheids.

Observational method and specific remark.

(1) Photoelectric scanner. No clear correlation was found for A supergiants.

(2) Photoelectric scanner.

(3) Narrow-band photometry.

(4) Photographic spectroscopy (16 or $\left.33 \AA \mathrm{mm}^{-1}\right)$. $E \equiv W(\mathrm{O}$ I $7771-5) \times W($ Fe I 7748$)$.

(5) Spectroscopy with linear photodiode array $\left(10 \AA \mathrm{mm}^{-1}\right)$.

(6) Spectroscopy with Reticon $\left(50 \AA \mathrm{mm}^{-1}\right)$.

(7) Narrow-band photometry. $W_{77}=20.8 \Lambda(16)-44.3$.

(8) High-resolution CCD spectroscopy $\left(8 \AA \mathrm{mm}^{-1}\right)$.

(9) Narrow-band photometry and low-resolution spectroscopy.

(10) Narrow-band photometry and CCD spectroscopy. Application of Arellano Ferro \& Mendoza (1993) formula to derive $M_{V}$.

(11) Medium-resolution CCD spectroscopy.

(12) Medium-resolution CCD spectroscopy.

(13) High-resolution CCD spectroscopy $(R=18000)$.

(14) High-dispersion spectroscopy $(R=52000$ or 85000$)$. Their Eq.(2) is an intricate relation in terms of $T_{\text {eff }}, \log g, \xi$, and [Fe/H].

(15) Use of published $W_{77}$ data from various literature. 
Table 2. Basic stellar parameters and the results of equivalent widths as well as microturbulences for 75 program stars.

\begin{tabular}{|c|c|c|c|c|c|c|c|c|c|c|c|c|}
\hline $\begin{array}{c}\mathrm{HD \#} \\
(1)\end{array}$ & $\begin{array}{c}\text { Name } \\
(2)\end{array}$ & $\begin{array}{c}\text { Sp. Type } \\
\text { (3) }\end{array}$ & $\begin{array}{l}M_{V} \\
(4) \\
\end{array}$ & $\begin{array}{c}\log L \\
(5)\end{array}$ & $\begin{array}{l}M \\
(6) \\
\end{array}$ & $\begin{array}{l}T_{\text {eff }} \\
(7)\end{array}$ & $\begin{array}{c}\log g \\
(8) \\
\end{array}$ & $\begin{array}{l}v_{\mathrm{M}} \\
(9) \\
\end{array}$ & $\begin{array}{c}\xi_{\mathrm{p}} \\
(10) \\
\end{array}$ & $\begin{array}{l}W_{77} \\
(11)\end{array}$ & $\begin{array}{c}\xi_{\mathrm{a}} \\
(12) \\
\end{array}$ & $\begin{array}{l}\text { Remark } \\
(13)\end{array}$ \\
\hline 000571 & 22 And & F2 II & -3.25 & 3.202 & 5.42 & 6995 & 2.30 & 55 & 6.0 & 0.969 & 5.7 & \\
\hline 006130 & & FO II & -4.04 & 3.519 & 6.54 & 7616 & 2.22 & 17 & 4.9 & 1.035 & 4.8 & \\
\hline 006210 & & $\mathrm{~F} 6 \mathrm{~V}$ & +1.19 & 1.451 & 1.97 & 5983 & 3.34 & 46 & 8.5 & 0.405 & & \\
\hline 007034 & $82 \mathrm{Psc}$ & Fo V & -0.82 & 2.229 & 2.97 & 7349 & 3.10 & 109 & 4.1 & 0.917 & 4.4 & \\
\hline 008890 & $\alpha \mathrm{UMi}$ & F7: Ib-IIv SB & -3.73 & 3.428 & 6.50 & 5741 & 1.81 & 13 & 5.5 & 0.759 & 4.4 & Cepheid \\
\hline 012953 & & A1 Ia & -6.43 & 4.502 & 12.83 & 9475 & 1.90 & 40 & 18.4 & 1.852 & 14.2 & \\
\hline 014489 & 9 Per & A2 Ia & -5.07 & 3.949 & 8.54 & 9185 & 2.23 & 30 & 15.5 & 1.686 & 11.1 & 1st Hipp. plx \\
\hline 020902 & $\alpha$ Per & F5 Ib & -4.34 & 3.650 & 7.35 & 6389 & 1.83 & 20 & 6.5 & 1.094 & 5.6 & \\
\hline 022211 & & G0 & +0.77 & 1.633 & 2.27 & 5720 & 3.14 & 96 & 6.4 & 0.576 & & \\
\hline 023230 & 41 Per & F5 IIvar & -2.64 & 2.960 & 4.71 & 6728 & 2.42 & 46 & 5.3 & 0.857 & 4.3 & double line? \\
\hline 025291 & & F0 II & -4.63 & 3.755 & 7.65 & 7677 & 2.06 & 11 & 3.8 & 1.059 & 4.9 & 1st Hipp. plx \\
\hline 026553 & & A4 III & -2.89 & 3.059 & 5.00 & 6767 & 2.35 & 9 & 2.9 & 0.945 & 3.2 & \\
\hline 032188 & & A2sh & -3.42 & 3.287 & 5.47 & 8976 & 2.66 & 30 & 2.5 & 0.875 & 3.5 & \\
\hline 032655 & & F2 IIp... & -0.85 & 2.248 & 3.08 & 6548 & 2.90 & 30 & 5.0 & 0.927 & 4.5 & \\
\hline 035520 & & A1p & -2.78 & 3.049 & 4.69 & 9763 & 2.97 & 97 & 2.4 & 0.804 & 3.5 & 1st Hipp. plx \\
\hline 038529 & & $\mathrm{G} 4 \mathrm{~V}$ & +2.95 & 0.790 & 1.46 & 5320 & 3.67 & 7 & 1.5 & 0.225 & 0.0 & \\
\hline 038558 & $130 \mathrm{Tau}$ & F0 III & -3.58 & 3.333 & 5.80 & 7579 & 2.34 & 26 & 4.7 & 1.019 & 5.0 & \\
\hline 039866 & & A2 II & -5.13 & 4.000 & 8.73 & 10212 & 2.37 & 13 & 3.7 & 1.017 & 4.6 & 1st Hipp. plx \\
\hline 045412 & 48 Aur & F5.5 Ibv & -3.11 & 3.186 & 5.60 & 5694 & 1.98 & 13 & 6.0 & 0.905 & 4.7 & Cepheid, 1st Hipp. plx \\
\hline 050018 & 59 Aur & F2 V & +0.07 & 1.879 & 2.51 & 6660 & 3.21 & 155 & & 0.814 & 2.8 & \\
\hline 050420 & & A9 III & -1.53 & 2.511 & 3.56 & 7229 & 2.87 & 29 & 5.4 & 0.812 & 4.0 & \\
\hline 057749 & & F3 IV & -1.05 & 2.325 & 3.21 & 6803 & 2.90 & 43 & 3.4 & 0.775 & 3.0 & \\
\hline 059881 & $\delta^{1} \mathrm{CMi}$ & F0 III & -1.62 & 2.550 & 3.63 & 7332 & 2.86 & 60 & 4.1 & 1.002 & 4.4 & \\
\hline 072779 & $35 \mathrm{Cnc}$ & GO III & -0.05 & 1.967 & 2.78 & 5596 & 2.86 & 96 & 8.3 & 0.614 & 10.0 & \\
\hline 077601 & & F6 II-III & +0.53 & 1.707 & 2.30 & 6216 & 3.22 & 141 & 4.8 & 0.870 & 6.9 & \\
\hline 082210 & $24 \mathrm{UMa}$ & G4 III-IV & +2.00 & 1.173 & 1.88 & 5294 & 3.39 & 5 & 4.8 & 0.157 & $\ldots$ & \\
\hline 082543 & & F7 IV-V & +0.76 & 1.633 & 2.26 & 5742 & 3.15 & 6 & 4.9 & 0.273 & $\ldots$ & \\
\hline 088759 & & $\mathrm{~F} 2$ & +0.35 & 1.770 & 2.36 & 6518 & 3.25 & 90 & 7.0 & 0.652 & 7.8 & \\
\hline 100418 & & F8/G0 Ib/II & +0.53 & 1.721 & 2.39 & 5847 & 3.12 & 35 & 6.2 & 0.492 & 2.0 & \\
\hline 104452 & $1 \mathrm{Com}$ & G0 II & +0.59 & 1.710 & 2.41 & 5608 & 3.06 & 55 & 8.4 & 0.430 & $\ldots$ & \\
\hline 111812 & $31 \mathrm{Com}$ & G0 III & +0.15 & 1.889 & 2.69 & 5554 & 2.91 & 64 & 7.8 & 0.446 & $\ldots$ & \\
\hline 111892 & 33 Com & F8 & +1.43 & 1.357 & 1.89 & 5903 & 3.40 & 37 & 5.0 & 0.426 & 1.2 & \\
\hline 117566 & & G2.5 IIIb & +1.00 & 1.569 & 2.34 & 5327 & 3.10 & 10 & 5.0 & 0.256 & 2.2 & \\
\hline 126868 & $\phi$ Vir & G2 III & +2.00 & 1.154 & 1.79 & 5511 & 3.46 & 15 & 5.3 & 0.262 & & \\
\hline 128563 & & $\mathrm{~F} 8$ & +2.05 & 1.109 & 1.63 & 5927 & 3.59 & 26 & 13.9 & 0.417 & 0.3 & sharp component \\
\hline 133002 & & F9 V & +2.41 & 0.985 & 1.58 & 5599 & 3.60 & 7 & 2.3 & 0.175 & $\ldots$ & \\
\hline 141714 & $\delta \mathrm{CrB}$ & G5 III-IV & +0.96 & 1.590 & 2.38 & 5284 & 3.07 & 4 & 4.8 & 0.173 & $\ldots$ & \\
\hline 148317 & & G0 III & +2.17 & 1.076 & 1.66 & 5649 & 3.54 & 6 & 2.5 & 0.309 & 0.3 & \\
\hline 148743 & & A7 Ib & -4.75 & 3.800 & 7.91 & 7581 & 2.01 & 20 & 6.0 & 1.536 & 7.2 & \\
\hline 149662 & & $\mathrm{~F} 2$ & +1.54 & 1.300 & 1.80 & 6192 & 3.52 & 141 & 2.6 & 0.865 & 4.3 & \\
\hline 151043 & & $\mathrm{~F} 8$ & +2.23 & 1.049 & 1.61 & 5713 & 3.58 & 10 & 3.8 & 0.323 & 0.0 & \\
\hline 151070 & & F5 III & +0.87 & 1.578 & 2.15 & 5977 & 3.25 & 23 & 2.9 & 0.394 & $\ldots$ & double line? \\
\hline 154319 & & K0 & +1.64 & 1.301 & 1.98 & 5445 & 3.33 & 7 & 1.9 & 0.275 & 1.1 & \\
\hline 158170 & & F5 IV & $\begin{array}{r}1.24 \\
\end{array}$ & 1.426 & 1.94 & 6069 & 3.39 & 7 & 4.8 & 0.586 & 2.4 & \\
\hline 159026 & & F6 III & -0.99 & 2.313 & 3.25 & 6174 & 2.75 & 156 & & 0.889 & 4.8 & \\
\hline 159877 & & F0 IV & -3.77 & 3.441 & 5.98 & 9676 & 2.67 & 24 & 3.9 & 1.002 & 5.7 & \\
\hline 160365 & & F6 III & +0.93 & 1.550 & 2.09 & 6083 & 3.30 & 102 & & 0.695 & 9.3 & \\
\hline 164136 & $\nu$ Her & F2 II & -2.82 & 3.032 & 4.92 & 6738 & 2.37 & 32 & 5.7 & 0.766 & 4.5 & \\
\hline 164507 & & G5 IV & +2.95 & 0.780 & 1.43 & 5429 & 3.71 & 6 & 1.8 & 0.196 & 0.2 & \\
\hline 164613 & $\psi^{2}$ Dra & F2.5 II-III & -2.21 & 2.785 & 4.21 & 6921 & 2.59 & 43 & 5.3 & 0.887 & 4.3 & \\
\hline 164668 & 95 Her B & G5 & -0.45 & 2.179 & 3.37 & 5027 & 2.55 & 6 & 4.8 & 0.223 & $\ldots$ & $V$ and $B-V$ from simbad \\
\hline 168608 & Y Sgr & F8 II & -3.25 & 3.201 & 5.43 & 6887 & 2.28 & 18 & 7.1 & 0.767 & 4.5 & Cepheid \\
\hline 172365 & & F8 Ib-II & -2.10 & 2.768 & 4.32 & 5972 & 2.36 & 52 & 7.0 & 0.771 & 5.2 & \\
\hline 174464 & & $\mathrm{~F} 2 \mathrm{Ib}$ & -3.67 & 3.368 & 5.96 & 7403 & 2.28 & 22 & 5.0 & 1.013 & 5.1 & 1st Hipp. plx \\
\hline 180583 & V473 Lyr & F6 Ib-II & -2.52 & 2.925 & 4.69 & 6253 & 2.32 & 12 & 5.4 & 0.743 & 5.3 & Cepheid \\
\hline 185758 & $\alpha$ Sge & G0 II & -1.33 & 2.494 & 3.83 & 5400 & 2.41 & 7 & 4.9 & 0.322 & 1.5 & \\
\hline 186377 & & A5 III & -3.56 & 3.357 & 5.67 & 9590 & 2.72 & 13 & 3.5 & 0.978 & 4.6 & \\
\hline 187982 & & A1 Iab & -4.43 & 3.674 & 7.28 & 7413 & 2.06 & 28 & 15.5 & 1.906 & 9.7 & \\
\hline 188650 & & $\mathrm{Fp}$ & -2.44 & 2.921 & 4.80 & 5645 & 2.16 & 11 & 5.7 & 0.547 & 8.8 & \\
\hline 192514 & 30 Cyg & A5 IIIn & -1.75 & 2.610 & 3.67 & 8507 & 3.07 & 175 & 2.7 & 0.974 & 4.9 & \\
\hline 193370 & 35 Cyg & F5 Ib & -4.32 & 3.648 & 7.39 & 6149 & 1.77 & 13 & 5.4 & 1.082 & 6.2 & 1st Hipp. plx \\
\hline 194951 & & F1 II & -3.83 & 3.434 & 6.21 & 7418 & 2.23 & 22 & 5.2 & 1.126 & 5.2 & 1st Hipp. plx \\
\hline 195295 & 41 Cyg & F5 II & -2.96 & 3.092 & 5.13 & 6624 & 2.29 & 11 & 5.0 & 0.963 & 4.9 & \\
\hline 195324 & 42 Cyg & A1 Ib & -4.87 & 3.862 & 8.06 & 8849 & 2.22 & 21 & 6.3 & 1.405 & 7.4 & \\
\hline 196755 & $\kappa$ Del & G5 IV $+\ldots$ & +2.67 & 0.889 & 1.51 & 5482 & 3.64 & 7 & 2.1 & 0.213 & 1.5 & \\
\hline 197345 & $\alpha$ Cyg & A2 Ia & -7.65 & 5.006 & 18.33 & 10188 & 1.68 & 31 & 14.1 & 1.928 & 11.8 & \\
\hline 198726 & $\mathrm{~T}$ Vul & F5 Ib & -2.44 & 2.902 & 4.68 & 5974 & 2.27 & 15 & 6.6 & 0.904 & 4.7 & Cepheid \\
\hline 201078 & DT Cyg & F7.5 Ib-IIv & -3.15 & 3.173 & 5.44 & 6339 & 2.16 & 13 & 5.1 & 0.782 & 3.6 & Cepheid \\
\hline 202240 & & F0 III & -3.02 & 3.128 & 4.96 & 8952 & 2.77 & 17 & 4.1 & 1.029 & 4.9 & \\
\hline 203096 & & A5 IV & -3.35 & 3.247 & 5.41 & 8251 & 2.54 & 28 & 4.1 & 0.987 & 5.0 & \\
\hline 208110 & & G0 IIIs & +0.36 & 1.828 & 2.70 & 5309 & 2.89 & 6 & 2.4 & 0.136 & & \\
\hline 210459 & $\pi \mathrm{Peg}$ & F5 III & -0.30 & 2.035 & 2.78 & 6262 & 2.99 & 146 & 5.9 & 0.867 & 5.5 & \\
\hline 213306 & $\delta$ Cep & G2 Ibvar & -3.66 & 3.393 & 6.32 & 5890 & 1.88 & 14 & 5.2 & 0.536 & 2.9 & Cepheid \\
\hline 218753 & 2 Cas & A5 III & -4.10 & 3.567 & 6.52 & 9395 & 2.53 & 9 & 2.8 & 0.988 & 4.5 & \\
\hline 220657 & $v$ Peg & F8 IV & +0.79 & 1.621 & 2.28 & 5754 & 3.17 & 74 & 7.5 & 0.476 & $\ldots$ & \\
\hline
\end{tabular}

(1) HD number. (2) Bayer/Flamsteed name. (3) Spectral type taken from Hipparcos catalogue (ESA 1997). (4) Extinction-corrected absolute visual magnitude (in mag). (5) Logarithmic bolometric luminosity $\log \left(L / L_{\odot}\right)$ (in dex), where $L_{\odot}$ is the solar luminosity. (6) Stellar mass (in $M_{\odot}$ ). (7) Effective temperature (in K). (8) Logarithm of surface gravity $\log g$ (in dex), where $g$ is in unit of $\mathrm{cm} \mathrm{s}^{-2}$. (9) Macrobroadening velocity in $\mathrm{km} \mathrm{s}^{-1}$ (nearly equivalent to the projected rotational velocity $v_{\mathrm{e}} \sin i$ for large $v_{\mathrm{M}}$, though the contribution of macroturbulence may be significant for the case of small $v_{\mathrm{M}}$ with $\lesssim$ several tens $\mathrm{km} \mathrm{s}^{-1}$ ). (10) Profile-based microturbulence (in $\mathrm{km} \mathrm{s}^{-1}$ ). (11) Equivalent width of the whole O I 7771-5 triplet (in $\AA$ ). (12) Abundance-based microturbulence (in km s${ }^{-1}$ ). (13) Specific remark. The notation "1st Hipp. plx"means that the parallax data was taken from the first version of the Hipparcos catalogue (ESA 1997), instead of the revised new reduction data (van Leeuwen 2007). 
Table 3. Adopted atomic data of oxygen lines.

\begin{tabular}{|c|c|c|c|c|c|c|c|}
\hline Line & $\begin{array}{l}\text { Equivalent } \\
\text { Width }\end{array}$ & $\begin{array}{l}\lambda \bar{\lambda} \\
(\AA)\end{array}$ & $\begin{array}{l}\chi_{\text {low }} \\
(\mathrm{eV})\end{array}$ & $\begin{array}{l}\log g f \\
(\operatorname{dex})\end{array}$ & $\begin{array}{c}\text { Gammar } \\
\text { (dex) }\end{array}$ & $\begin{array}{c}\text { Gammas } \\
\text { (dex) }\end{array}$ & $\begin{array}{c}\text { Gammaw } \\
\text { (dex) }\end{array}$ \\
\hline \multirow[t]{3}{*}{ O г $7771-5$} & $W_{77}$ & 7771.944 & 9.146 & +0.324 & 7.52 & -5.55 & $(-7.65)$ \\
\hline & (3 components) & 7774.166 & 9.146 & +0.174 & 7.52 & -5.55 & $(-7.65)$ \\
\hline & & 7775.388 & 9.146 & -0.046 & 7.52 & -5.55 & $(-7.65)$ \\
\hline \multirow[t]{9}{*}{ O г $6155-8$} & $W_{61}$ & 6155.961 & 10.740 & -1.401 & 7.60 & -3.96 & $(-7.23)$ \\
\hline & (9 components) & 6155.971 & 10.740 & -1.051 & 7.61 & -3.96 & $(-7.23)$ \\
\hline & & 6155.989 & 10.740 & -1.161 & 7.61 & -3.96 & $(-7.23)$ \\
\hline & & 6156.737 & 10.740 & -1.521 & 7.61 & -3.96 & $(-7.23)$ \\
\hline & & 6156.755 & 10.740 & -0.931 & 7.61 & -3.96 & $(-7.23)$ \\
\hline & & 6156.778 & 10.740 & -0.731 & 7.62 & -3.96 & $(-7.23)$ \\
\hline & & 6158.149 & 10.741 & -1.891 & 7.62 & -3.96 & $(-7.23)$ \\
\hline & & .172 & 10.741 & -1.031 & 7.62 & -3.96 & $(-7.23)$ \\
\hline & & .187 & 10.741 & -0.441 & 7.61 & -3.96 & $(-7.23)$ \\
\hline
\end{tabular}

Following columns 3-5 (laboratory wavelength, lower excitation potential, and statistical weight of lower level times oscillator strength), damping parameters are presented in columns 6-8: Gammar is the radiation damping width $\left(\mathrm{s}^{-1}\right)$ [ $\left.\log \gamma_{\mathrm{rad}}\right]$, Gammas is the Stark damping width $\left(\mathrm{s}^{-1}\right)$ per electron density $\left(\mathrm{cm}^{-3}\right)$ at $10^{4} \mathrm{~K}\left[\log \left(\gamma_{\mathrm{e}} / N_{\mathrm{e}}\right)\right]$, and Gammaw is the van der Waals damping width $\left(\mathrm{s}^{-1}\right)$ per hydrogen density $\left(\mathrm{cm}^{-3}\right)$ at $10^{4} \mathrm{~K}\left[\log \left(\gamma_{\mathrm{w}} / N_{\mathrm{H}}\right)\right]$.

These data were taken from the compilation of Kurucz and Bell (1995) as long as available, while the parenthesized damping parameters are the default values computed by the WIDTH9 program. 